\title{
LA GEOGRAFÍA EN LA LOMCE ¿UNA OCASIÓN PERDIDA?
}

\author{
$M^{a}$ Ángeles Rodríguez Domenech \\ Departamento de Geografía y Ordenación del Territorio. Universidad de Castilla-La Mancha \\ Mangeles.Rodriguez@uclm.es
}

\section{RESUMEN}

La reciente reforma del Sistema Educativo Español llevada a cabo a través de la LOMCE en diciembre de 2013, conlleva cambios curriculares que afectan a las distintas disciplinas que se imparten en los diferentes niveles educativos y es una ocasión propicia para la reflexión sobre aquello que se puede y debe cambiar en cada una de ellas. La inminente implantación de la misma para el curso 2014-2015 en Educación Primaria y en la Formación Profesional básica, hacen que en este contexto, y referido a la Geografía, se analice en qué medida las carencias detectadas en la enseñanza de los conocimientos geográficos en la ley vigente (LOE) se han tenido en cuenta en los currículos propuestos en la nueva ley, o si, por el contrario, se persiste en las deficiencias haciendo de la reforma una ocasión perdida para conseguir su mejora.

Palabras clave: Reforma Educativa, LOE, LOMCE, Geografía, Educación Secundaria Obligatoria, Bachillerato y Formación Profesional.

\section{ABSTRACT}

The recent reform of the Spanish Educational System which has been accomplished through the OLIEQ in December 2013, entails curricular changes which concern the different disciplines which are taught in the existing educational levels and it is a favorable chance to reflect on what can be changed and must be changed in the aforesaid disciplines. The imminent establishment of this organic law in the academic year 2014-2015 in Primary Education and Basic Vocational Training make us analse to what extent the detected gaps

Fecha de recepción: febrero 2014.

Fecha de aceptación: enero 2015. 
in geographical knowledge teaching in the current law (Organic Law of Education - OLE) in this context and in reference to Geography have been taken into account in the suggested curriculum in the new law or if, on the contrary, it is insisted on the deficiencies to make the reform be a lost chance to achieve its progress.

Key words: Educational Reform, OLE (Organic Law of Education), OLIEQ (Organic Law for the Improvement of the Educational Quality), Geography, Compulsory Secondary Education and Vocational Training.

\section{INTRODUCCIÓN}

La reciente reforma del Sistema Educativo Español (en adelante (SEE) llevada a cabo a través de la Ley Orgánica 8/2013, de 9 de diciembre, para la mejora de la calidad educativa (en adelante LOMCE) y que se implantó en el curso académico 2014-2015 para la Educación Primaria y Formación Profesional Básica, y para el 2015-2016 para la Educación Secundaria Obligatoria (en adelante E.S.O.) y el Bachillerato, nos plantea una necesaria reflexión sobre los contenidos que esta normativa nos ofrece. Toda reforma educativa suele conllevar, como no podía ser menos, cambios curriculares de las distintas áreas de conocimiento que la integran. Despertando la consiguiente inquietud en los investigadores y docentes de todos los niveles de las mismas, derivada del deseo de conocer cómo queda configurada, desde el punto de vista normativo, su disciplina. Se tiene el deseo y justa aspiración de que se recoja en ella el estado actual de los conocimientos del área correspondiente, la toma en consideración de los planteamientos didácticos que se hayan producido hasta el momento y hayan demostrado su eficacia, y que su encuadre en el sistema permita alcanzar los objetivos educativos que le son propios.

En esta línea, la Asociación de Geógrafos Españoles (en adelante AGE) y el Colegio de Geógrafos en el tiempo que ha mediado desde el anuncio de una nueva ley de educación, hasta que han sido presentados los proyectos normativos para desarrollar dicha ley, pasando por su tramitación parlamentaria y su promulgación, han elaborado, publicado y presentado a los responsables políticos algunos documentos en los que se ha puesto de manifiesto el papel que la Geografía ha de desempeñar en la educación. Estos, sin embargo, han estado cercenados, en gran medida, porque no han sido las propuestas que les hubiera gustado presentar sino meras correcciones y sugerencias a las propuestas oficiales del Ministerio de Educación, Cultura y Deporte (en adelante (MECD), situación que ha generado un gran condicionamiento para la AGE. No obstante es de valorar el esfuerzo y los resultados obtenidos por este colectivo. En tal sentido se han hecho públicas tanto las deficiencias y carencias que, a su juicio, encontraron en el proyecto de ley antes y durante su tramitación parlamentaria; como a los proyectos de Real Decreto (en adelante RD) presentado por el MECD para establecer el currículo básico en Educación Primaria, Formación Profesional Básica, Secundaria y Bachillerato, a la vez que han hecho una serie de propuestas para mejorar dichos proyectos de «currículos» ${ }^{1}$ que no siempre han sido tenidos en cuenta. Trabajos que, junto con su experiencia y saber, se ha apoyado en una encuesta a geógrafos hecha al objeto de recabar su opinión

1 Alegaciones AGE al proyecto de R.D sobre los currículos de E.S.O. y del Bachillerato. Análisis y alegaciones de la AGE al Proyecto de Real Decreto sobre los currículos de E.S.O. (AGE. Noticias 10/01/2014). 
e inquietudes, con la finalidad de que esas conclusiones traspasasen el ámbito puramente profesional y sirviesen para aportar luces al legislador en la expresión normativa de la disciplina.

Con el fin de contribuir a este objetivo nos proponemos analizar en este trabajo (enero 2015), desde la perspectiva de la Geografía, primero, cuáles han sido las razones que han llevado a plantear una reforma del SEE, así como los avatares que ha seguido este anteproyecto de ley hasta convertirse en ley (Ley Orgánica 8/2013, LOMCE) y los RDs que la desarrollan.

En segundo lugar, nos proponemos plantear las principales diferencias entre la situación de la Geografía en la ley vigente (Ley Orgánica de Educación 2/2006, en adelante LOE) y la nueva ley (LOMCE), con la advertencia de que tal comparación se hace en base a los RDs ya publicados por el MECD para desarrollar la ley (RD 126/2014 por el que se establece el currículo básico de la Educación Primaria, RD 127/2014 para la Formación Profesional Básica, y RD 1105/2014 para la Educación Secundaria Obligatoria (E.S.O) y el Bachillerato, nos anticipa cual es la función formativa, los contenidos y el tiempo asignado en el currículo básico que se prevé para la Geografía en la nueva ley.

Y en tercero y último lugar, analizamos en qué medida se han rectificado o perpetuado las carencias que los conocimientos geográficos tienen en la LOE, a la vez que hacemos algunas propuestas de mejora que, a nuestro juicio, deben producirse en el encuadre normativo de la Geografía cómo disciplina en la enseñanza no universitaria. Nos apoyamos para ello tanto en los numerosos trabajos publicados por la AGE, como en los publicados por algunos profesores de Geografía en distintos niveles educativos, así como en nuestra propia reflexión y experiencia.

La metodología utilizada ha sido comparar tanto los textos de ambas leyes, LOMCE y LOE, o mejor las modificaciones que la LOMCE hace a la LOE, circunscribiendo esta comparación a la situación de la Geografía; centrándonos en los RDs publicados por el MECD para desarrollar la LOMCE en Educación Primaria (RD 126/2014 de 28 de febrero), en la Formación Profesional Básica (RD 127/2014 de 28 de febrero) y en E.S.O y Bachillerato (RD 1105/2014 de 26 de diciembre) que desarrollan la LOMCE en lo que respecta al Estado, sin entrar en las particularidades de cada Comunidad Autónoma.

\section{LA JUSTIFICACIÓN Y LOS AVATARES DE LA LOMCE COMO INSTRUMENTO DE REFORMA DEL SISTEMA EDUCATIVO ESPAÑOL (SEE)}

El Gobierno español promulgó en diciembre de 2013 la LOMCE, también denominada «Ley Wert», que se presenta técnicamente como una reforma de la LOE (2006) y de la LOGSE (1990), leyes estas últimas que regulaban, hasta el momento, el SEE.

La justificación de la nueva ley se apoya, según el Ministro, en dos hechos fundamentales:

1. Los informes internacionales sobre el SEE que sitúan a nuestro país en uno de los últimos lugares de los países analizados.

2. En el alto índice de fracaso escolar que genera fuertes desigualdades sociales: «no [se] puede asumir como normal o estructural que una parte importante de sus alumnos, aquellos que abandonan las aulas antes de disponer de los conocimientos, competencias y habilidades básicos, o aquellos cuyo nivel formativo esté muy por debajo de los estándares de calidad internacionales, partan en el inicio de su vida laboral en unas condiciones de desventaja tales que estén abocados al 
desempleo o a un puesto de trabajo de limitado valor añadido» (Boletín Oficial de las Cortes Generales. Congreso de los Diputados. nº 48-4, pág. 3).

Nuestro objetivo, como se indicó antes, no es analizar la LOMCE ni la normativa de RDs que la desarrollan en su conjunto, sino centrarnos en la configuración que se le da en estas normas legales a nuestra disciplina, la Geografía. Sin embargo, entendemos que debemos analizar con carácter general y como marco de referencia, tanto las razones que se han esgrimido para justificar la promulgación de una nueva ley de educación, como conocer si los cambios habidos en los diferentes textos legislativos han afectado a la situación de la Geografía.

\section{II.1. Los informes sobre el SEE como justificación de la LOMCE}

Los informes internacionales de educación, como es de sobra conocido, son informes realizados a nivel mundial o de grandes espacios que se basan en el análisis del rendimiento de los alumnos, de los profesores y de otros factores, y permiten hacer comparaciones de los distintos sistemas educativos.

Existe una gran variedad de informes realizados: unos por la OCDE (Organización para la Cooperación y el Desarrollo Económico $)^{2}$, otros por la $\mathrm{UNESCO}^{3}$, otros por la $\mathrm{UE}^{4}$ y otros por consultoras privadas 5 .

En España se encarga de hacer estos estudios el Instituto Nacional de Evaluación Educativa que es el organismo responsable de la evaluación del sistema educativo en el MECD.

Teniendo en consideración los datos de estos análisis, el MECD presentó, junto con los distintos borradores de la LOMCE, un documento: Propuestas para la Mejora de la Calidad Educativa (17 de mayo 2013) en el que analiza las debilidades y fortalezas del SEE.

Entre las fortalezas del sistema señala: 1) El gasto público por alumno en la educación pública en España es superior, en un 21\%, a la media de los países de la OCDE y de la UE; 2) En la última década se ha duplicado el gasto en educación no universitaria; 3) El número de alumnos por profesor es más bajo que en la OCDE; y 4) El gasto de España en la educación infantil (para las instituciones públicas) es superior al de la OCDE.

Las debilidades que estos documentos indican son, en términos generales, las altas tasas de fracaso escolar; el incremento del número de jóvenes que ni estudian ni trabajan; y las diferencias de rendimiento entre las distintas autonomías.

2 Son de distinto tipo según se realicen las pruebas al alumnado (PIRLS: Estudio Internacional de Progreso en Comprensión Lectora), TIMS (Estudio Internacional de Tendencias en Matemáticas y Ciencias), ICCS) o a los profesores (TEDS-M, TALIS). El informe PISA (Program for International Student Assessment o Informe del Programa internacional para la Evaluación de Estudiantes) por su parte, es una prueba que se realiza cada tres años a los alumnos de 15 años. Tiene como fin la valoración internacional de los alumnos a través de pruebas estandarizadas de tres áreas de conocimiento (Lectura, Matemáticas y Ciencias).

3 El Informe \& Educación para todos (EPT) parte de los informes y datos cuantitativos que ofrecen los distintos gobiernos.

4 Es el EECL (Estudio Europeo de Competencia Lingüística EECL).

5 Entre los informes privados queremos destacar: Educación en España, motivos para la esperanza publicado en enero de 2012 por la consultora McKinsey \& Company; El prestigio de la profesión docente. Percepción y realidad, realizado por la Fundación Europea Sociedad y Educación (EFSE), en colaboración con la Fundación Botín; y Educación y Desarrollo. Pisa 2009 y el sistema educativo español, elaborado por el Instituto Valenciano de Investigaciones Económicas (IVIE) y la Fundación BBVA. 
Debilidades que en el documento del MECD se concretan en los siguientes puntos: 1) La tasa de titulados en educación Secundaria, entre los 25-34 años, en España es inferior a la de los países de la OCDE; 2) El porcentaje de población entre 20 y 24 años que ha completado, al menos, la Educación Secundaria superior es inferior en España con respecto a Europa y se ha acrecentado en la última década porque en España han seguido disminuyendo mientras han crecido en Europa; 3) El abandono temprano de la educación y de la formación en España duplica a la media de la UE; 4) La población entre 15-19 años que ni trabaja ni estudia (NI-NI) es superior en España; 5) La tasa de paro juvenil en España duplica a la de la UE estando este dato en relación tanto con el nivel de estudios alcanzado como con la situación de la Formación Profesional; 6) Falta de señalización o control externo del SEE; 7) Escasa autonomía de los centros docentes; y 8) Bajo nivel en lenguas extranjeras.

El MECD, en el documento de referencia, no hace mención, dentro de las debilidades, a las diferencias entre Comunidades Autónomas (en adelante CC.AA), hecho que se destaca en los informes internacionales y en algunos internos, tales como:

1. En los resultados educativos entre CC.AA. se dan diferencias tan grandes como las que se dan entre países de la OCDE. Estas diferencias en el rendimiento pueden equivaler hasta a un año y medio de escolarización. Concretamente, Castilla y León, Madrid y La Rioja tienen los mejores resultados, mientras que Ceuta y Melilla, Canarias, Baleares y Andalucía tienen los peores.

2. No hay una relación directa entre renta y educación, ya que todas las comunidades están por debajo de la media de la OCDE en términos de calidad, aunque algunas obtienen buenos resultados en rendimiento (Castilla y León, Madrid, Cataluña y La Rioja) y de equidad (País Vasco, Galicia y Cataluña).

Las conclusiones de los distintos tipos de informes sobre el SEE han hecho tomar conciencia a toda la sociedad española de la necesidad de la reforma del SEE vigente y ello en base a dos razones fundamentales:

1. El sistema de enseñanza comprensivo no ha dado los resultados esperados, debido, en opinión de algunos autores, a que «la generalización de una enseñanza de tipo comprehensivo que, entre otras cosas, supone, por un lado, atribuir un valor relativo al estricto aprendizaje del método científico como meta fundamental de la enseñanza y valorar más el cultivo de las vías psicológicas e intuitivas de aproximación a la realidad; y, de otro lado, la necesidad de conceder mayor importancia a los asuntos de actualidad a la hora de seleccionar temáticas» (Luis Gómez et al., 2006:333).

2. La universalización de la Enseñanza Secundaria como medida de igualdad y compensación social que presupone la atención a la diversidad y a las especificidades personales y ambientales de los alumnos» (Rodríguez Domenech, Ma A., 2008:45) hacen que «la escuela moderna sea la valedora de la educación como utopía de justicia social y bienestar» (Boletín Oficial de las Cortes Generales. Congreso de los Diputados. $n^{\circ} 48-4$, pág. 3), y por tanto hay que promover un sistema educativo eficaz. 


\section{II.2. De los diferentes borradores del anteproyecto de ley a la LOMCE}

Esta ley, además de haberse planteado como una reforma de la ley vigente (LOE, 2006) en lugar de como una ley nueva con todo el articulado, ha tenido diversas redacciones en su fase de Anteproyecto de Ley que se conocen como «borradores». La evolución temporal de los distintos borradores tiene especial interés a la hora de analizar los cambios que ha sufrido el tratamiento de la Geografía en las distintas versiones del anteproyecto de ley (Cuadro 1).

La primera de ellas o Borrador 1 (MECD, 25/09/2012) se elabora a partir de un documento presentado por el MECD el 11 de julio de 2012 titulado «Propuestas para el anteproyecto de Ley Orgánica para la Mejora de la Calidad Educativa» y de las sugerencias que los ciudadanos envían al correo electrónico habilitado por el MECD (calidadeducacion@ mecd.es).

El texto resultante entre las propuestas y las sugerencias se elevó al Consejo de Ministros por el Ministro de Educación el 21 de septiembre de 2012, con el fin de que este determinara los ulteriores trámites. A partir de ese momento se inician las consultas e informes (Conferencia Sectorial de Educación, la Conferencia General de Política Universitaria, el Consejo de Universidades, el Consejo Escolar del Estado, el Consejo Superior de Enseñanzas Artísticas, el Consejo General de la Formación Profesional y el Consejo de Estado.) para que hicieran las oportunas aportaciones. Entre estas cabe destacar, para nuestro objetivo, la realizada por la AGE (Buzo e Ibarra, 2013) y que más adelante tendremos ocasión de comentar.

Cuadro 1

FASES EN LA ELABORACIÓN DEL ANTEPROYECTO, DEL PROYECTO Y LA LEY 8/2013 (LOMCE)

\begin{tabular}{|c|c|c|}
\hline Fecha & Redacción o versión & Destino \\
\hline 09-07-2012 & $\begin{array}{c}\text { Propuestas para el } \\
\text { anteproyecto de LOMCE }\end{array}$ & Elaboración del texto del Anteproyecto de Ley \\
\hline 21-09-2012 & Versión 1. Primer borrador & $\begin{array}{c}\text { Presentación del Anteproyecto al Consejo de } \\
\text { Ministros a fin de que éste decida sobre los } \\
\text { ulteriores trámites. }\end{array}$ \\
\hline 19-12-2012 & Versión 2. Segundo borrador & $\begin{array}{c}\text { Texto remitido a Conferencia Sectorial de } \\
\text { Educación }\end{array}$ \\
\hline 12-02-2013 & Versión 3. Tercer borrador & $\begin{array}{c}\text { Texto remitido al Consejo de Estado. En el texto } \\
\text { se han incluido las aportaciones resultado de las } \\
\text { reuniones de la Conferencia de Educación del 4 } \\
\text { yel 19 de diciembre de 2012, y de la sesión del } \\
\text { Consejo Escolar del Estado del 24 de enero de } \\
\text { 2013 }\end{array}$ \\
\hline 17-05-2013 & Versión 4. Texto definitivo & Texto enviado al Congreso \\
\hline 24-05-2013 & Texto del proyecto de ley & $\begin{array}{c}\text { Publicación en Boletín Oficial de las Cortes } \\
\text { (8-X-2013) como Proyecto de Ley }\end{array}$ \\
\hline $09-12-2013$ & Texto de la ley & BOE no 295, pp.97858 \\
\hline
\end{tabular}

Fuente: Elaboración propia con datos del MECD. 
El Borrador 2 (MECD, 3/12/2012) se presenta el 19 de diciembre de ese mismo año, con modificaciones importantes con respecto al anterior, y el documento resultante fue remitido a la Conferencia Sectorial de Educación quien se reunió para estudiarlo entre el 4 y el 19 de diciembre de 2012. Este borrador es el dictaminado por el Consejo Escolar del Estado en su sesión del 24 de enero de 2013 con dictamen 1/2013.

El Borrador 3 (MECD, 14/02/2013) es el resultado de la toma en consideración tanto de las aportaciones de la Conferencia Sectorial como del Consejo Escolar del Estado y es este nuevo borrador el que se remite, el 24 de enero de 2013, para solicitar el dictamen del Consejo de Estado.

La tramitación previa culmina con la aprobación, el 17 de mayo de 2013, por el Consejo de Ministros del texto que se remite a las Cortes (Borrador 4. MECD, 8/10/2013) quien lo publica en el Boletín Oficial de las Cortes Generales el 24 de mayo de ese mismo año.

El texto del proyecto de ley comenzó a tramitarse en el Congreso después de que el 16 de julio de 2013 fueran rechazas 11 enmiendas a la totalidad. Posteriormente, terminado el trámite de enmiendas, en el que se presentaron un total de 1.666 enmiendas parciales (770 en el Congreso y 896 en el Senado), de las que fueron introducidas en el texto 67 del partido que apoya al gobierno y 40 de los grupos de la oposición, se aprobó, definitivamente, el 10 de octubre de 2013 en el Pleno del Congreso y el 20 de noviembre de ese mismo año en el pleno del Senado. Su publicación en el BOE tuvo lugar el 10 de diciembre de 2013.

\section{LOS CONTENIDOS GEOGRÁFICOS EN LA LOMCE Y SU DIFERENCIA CON LA LOE}

Los principales estudios evolutivos sobre el currículo de la Geografía en los niveles no universitarios han sido realizados por Souto $(2003,2004,2011)$ y por González Gallego $(2001,2011)$. En este trabajo, sin embargo, el análisis del currículo de la Geografía lo centramos entre la LOE y la reciente reforma del SEE a través de la LOMCE, y contemplando dos escalas: la de las propias leyes como marco de encuadre; y la de los RDs donde se desarrollan y determinan esos contenidos.

\section{III.1. La estructura del SEE en la LOMCE y sus diferencias con la LOE como marco de encuadre de la Geografía}

Antes de abordar la situación en que quedan los contenidos geográficos en los distintos niveles educativos de la LOMCE conviene tener presente algunos aspectos generales de la misma, así como los cambios que representa con respecto a la LOE. Entendemos que estas consideraciones nos ayudaran a centrar mejor la situación que se propone para la Geografía. Nos referimos a aspectos tales como la clasificación u organización de las distintas enseñanzas; lo que se entiende por «educación básica»; los elementos del currículo; y la organización de las diferentes etapas educativas.

1. En la LOMCE y en la LOE se mantiene la misma clasificación de las distintas enseñanzas que integran el SEE (Cuadro 2), así como el mismo concepto de educación básica, aunque en la LOMCE se hace una especial incidencia en la inclusión de la Formación Profesional Básica en el conjunto de la educación básica. 
Cuadro 2

COMPARACIÓN DE LA ORGANIZACIÓN DEL SEE EN LA LOE, EN LOS BORRADORES, EN EL PROYECTO DE LEY Y EN LALOMCE

\begin{tabular}{|c|c|c|c|c|c|c|}
\hline LOE & $1^{\circ}$ BORRADOR & $\begin{array}{c}2^{\circ} \\
\text { BORRADOR }\end{array}$ & $\begin{array}{c}3^{\circ} \\
\text { BORRADOR }\end{array}$ & $\begin{array}{c}\text { Proyecto de } \\
\text { ley }\end{array}$ & LOMCE & $\begin{array}{c}\text { VARIACIÓN } \\
\text { LOE } \\
\text { /LOMCE }\end{array}$ \\
\hline $\begin{array}{l}\text { 3. La educación } \\
\text { primaria y } \\
\text { la educación } \\
\text { Secundaria } \\
\text { Obligatoria } \\
\text { constituyen } \\
\text { la educación } \\
\text { básica. }\end{array}$ & $\begin{array}{c}\text { Tres. El apartado } 3 \\
\text { del artículo } 3 \text { queda } \\
\text { redactado de la siguiente } \\
\text { manera: } \\
\text { «3. La Educación } \\
\text { Primaria, la Educación } \\
\text { Secundaria Obligatoria y } \\
\text { los ciclos de Formación } \\
\text { Profesional Básica } \\
\text { constituyen la educación } \\
\text { básica.» }\end{array}$ & $\begin{array}{c}\text { Tres. Se añade un } \\
\text { nuevo apartado } 10 \\
\text { al artículo 3, con la } \\
\text { siguiente redacción: } \\
\text { «3. Los ciclos de } \\
\text { Formación Profesional } \\
\text { Básica tendrán carácter } \\
\text { obligatorio y } \\
\text { gratuito.» }\end{array}$ & $\begin{array}{l}\text { No tiene variación con } \\
\text { respecto al } 2^{\circ} \text { borrador }\end{array}$ & $\begin{array}{l}\text { Tres. Se añade un } \\
\text { nuevo apartado } 10 \\
\text { al artículo 3, con la } \\
\text { siguiente redacción: } \\
\text { «10. Los ciclos } \\
\text { de Formación } \\
\text { Profesional Básica } \\
\text { serán de oferta } \\
\text { obligatoria y } \\
\text { carácter gratuito» }\end{array}$ & $\begin{array}{c}\text { No tiene } \\
\text { variación } \\
\text { con respecto } \\
\text { al proyecto } \\
\text { de ley }\end{array}$ & $\begin{array}{l}\text { Queda como está } \\
\text { en LOE con la } \\
\text { incorporación del } \\
\text { apartado } 10\end{array}$ \\
\hline
\end{tabular}

Fuente: LOE, Borradores, Proyecto de ley y LOMCE. Elaboración propia.

Efectivamente, el capítulo III de la LOE y en su artículo 3, dedicado a las enseñanzas que componen el SEE, especifica los distintos tipos de enseñanza, tipos que se mantienen en la LOMCE dado que el precitado artículo mantiene el mismo texto aunque incorpora un nuevo punto, el 10, sobre la inclusión en este concepto de la Formación Profesional Básica. No obstante, conviene advertir que este planteamiento final del Proyecto de Ley no ha sido el mismo en las distintas fases que ha atravesado como anteproyecto.

2. Del conjunto de enseñanzas que integran el SEE en relación con la Geografía, nos interesa considerar la Educación Primaria, la E.S.O., el Bachillerato, y la Formación Profesional Básica por ser en las que, según la ley, se puede impartir nuestra disciplina, si bien más adelante haremos algunas consideraciones para su posible inclusión en otras enseñanzas (Cuadro 3).

3. Los planteamientos de la LOMCE y los de la LOE en Enseñanza Primaria son idénticos en lo que respecta a edad y número de cursos (de 5 a 12 años y 6 cursos), aunque en la LOMCE se contempla que los alumnos de Primaria realicen una doble prueba de evaluación: una al finalizar el tercer curso de Primaria, en la que se comprobará el grado de adquisición de las competencias básicas en comunicación lingüística y matemática (artículo 20.3), por lo que las Ciencias Sociales y con ellas la Geografía estarían excluidas; y otra al final de la etapa (artículo 21) en que parece que se continua con el mismo espíritu de exclusión de nuestra área.

La LOMCE en esta etapa (artículo 18), a diferencia de la LOE, no distingue ningún ciclo dentro de ella y se organiza en áreas que clasifica en troncales, específicas y de libre configuración, estando la Geografía incluida dentro de las primeras, es decir, en las troncales, si bien, desde nuestro punto de vista, cabe también incluir algún contenido geográfico entre las asignaturas de libre configuración que han de ser determinadas por las CC. Autónomas.

La LOE configuraba la Enseñanza Primaria en tres ciclos de dos cursos cada uno y estos en áreas semejantes, pero no diferencia entre troncales, específicas y de libre configuración. 


\begin{tabular}{|c|c|c|c|c|c|c|c|}
\hline \multicolumn{4}{|c|}{ LOE (2006) } & \multicolumn{4}{|c|}{ LOMCE (2013) } \\
\hline $\begin{array}{l}\text { Edad } \\
\text { (años) }\end{array}$ & Etapa & Cursos & Modalidad y ciclos & $\begin{array}{l}\text { Edad } \\
\text { (años) }\end{array}$ & Etapa & Cursos & Modalidad \\
\hline \multicolumn{4}{|c|}{$\begin{array}{l}\text { PRUEBA DE ACCE.S.O. A LA } \\
\text { UNIVERSIDAD }\end{array}$} & \multicolumn{4}{|c|}{$\begin{array}{l}\text { PRUEBA GENERAL DE FIN DE ETAPA } \\
\text { (REVÁLIDA) }\end{array}$} \\
\hline \multirow{3}{*}{$16-18$} & \multirow{3}{*}{ } & \multirow{3}{*}{$1^{\circ}$ y $2^{\circ}$} & Ciencias y Tecnología & \multirow{3}{*}{$16-18$} & \multirow{3}{*}{ 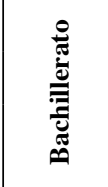 } & \multirow{3}{*}{$1^{\circ}$ y $^{\circ}$} & Ciencias \\
\hline & & & Artes & & & & Artes \\
\hline & & & \multirow[t]{5}{*}{$\begin{array}{l}\text { Humanidades y } \\
\text { CC. Sociales }\end{array}$} & & & & $\begin{array}{l}\text { Humanidades y } \\
\text { CC. Sociales }\end{array}$ \\
\hline \multirow{4}{*}{$12-16$} & \multirow{4}{*}{ 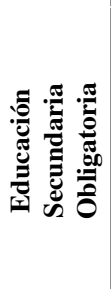 } & \multirow{4}{*}{$1^{\circ}$ a $4^{\circ}$} & & \multicolumn{4}{|c|}{$\begin{array}{l}\text { PRUEBA GENERAL DE FIN DE ETAPA } \\
\text { (REVÁLIDA) }\end{array}$} \\
\hline & & & & \multirow{3}{*}{$12-16$} & \multirow{3}{*}{ 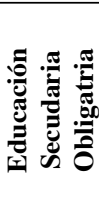 } & \multirow{2}{*}{$\begin{array}{c}4^{\circ} \\
\left(2^{\circ} \text { ciclo }\right)\end{array}$} & E. Académicas \\
\hline & & & & & & & $\begin{array}{l}\text { E. Aplicadas para la } \\
\text { iniciación de la F.P. }\end{array}$ \\
\hline & & & & & & $\begin{array}{l}1^{\circ}, 2^{\circ} \text { y } 3^{\circ} \\
\left(1^{\circ} \text { ciclo }\right)\end{array}$ & \\
\hline \multirow{3}{*}{$5-12$} & \multirow{3}{*}{ 婿 } & \multirow{3}{*}{$1^{\circ}$ a $6^{\circ}$} & $3^{\circ}$ ciclo $\left(4^{\circ}\right.$ y $\left.5^{\circ}\right)$ & \multicolumn{4}{|c|}{ PRUEBA GENERAL DE FIN DE ETAPA } \\
\hline & & & $2^{\circ}$ ciclo $\left(3^{\circ}\right.$ y $\left.4^{\circ}\right)$ & \multirow[t]{2}{*}{$5-12$} & \multirow{2}{*}{ 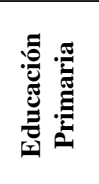 } & \multirow[t]{2}{*}{$1^{\circ}$ a $6^{\circ}$} & \multirow{2}{*}{$\begin{array}{l}\text { Las áreas pueden ser } \\
\text { - Troncales } \\
\text { - Específicas } \\
\text { - Libre } \\
\text { configuración }\end{array}$} \\
\hline & & & $1^{\circ}$ Ciclo $\left(1^{\circ}\right.$ y $\left.2^{\circ}\right)$ & & & & \\
\hline
\end{tabular}

Fuente: LOE, Borradores, Proyecto de ley, LOMCE y RDs. Elaboración propia.

No obstante, la diferencia más sustancial, desde nuestro punto de vista, es que la Geografía en la LOE está incluida en el área de Conocimiento del Medio Natural, Social y Cultural, mientras que en la LOMCE lo está en la de Ciencias Sociales, cambio que valoraremos más adelante.

4. La E.S.O. en los dos textos legislativos que comparamos sigue siendo igual en cuanto al número de cursos y la edad (4 cursos para alumnos entre 12 y 16 años) y difieren en cuanto que la LOMCE, establece dos ciclos (artículo 23bis): el primero, que comprende los tres primeros cursos; y el segundo ciclo que comprende el cuarto curso (artículos 23, 24 y 25), y tendrá, este último, un carácter propedéutico abriendo dos opciones: una Académica orientada hacia el Bachillerato, y otra de Enseñanzas para la iniciación de la Formación Profesional (F.P.). En ambas opciones se contempla el estudio de Ciencias Sociales.

Esta etapa está organizada en materias (artículo 24) que, a su vez, se clasifican en troncales y específicas, y dentro de las troncales, diferencia entre generales y de opción. Concretamente la Geografía, junto con la Historia, es considerada asignatura troncal general tanto en el primero como en el segundo ciclo de E.S.O. (articulo 13, RD 1105/2014). 
En la LOE no se diferencian ciclos ni hay opciones o modalidades, y las asignaturas forman un bloque de materias que tienen carácter obligatorio en los cuatro cursos y un número de asignaturas optativas que hay que elegir entre la oferta que se hace, a cursar a lo largo de la etapa.

También en esta Ley la Geografía se estudia en todos los cursos aunque con la designación de CC. Sociales, Geografía e Historia, mientras que en la LOMCE se la denomina Geografía e Historia. También se establece una Prueba General de Fin de Etapa en la que sí hay comprobación de adquisición de competencias en el caso de que haya sido elegida (artículo 29) (Cuadro 4).

Cuadro 4

LA GEOGRAFÍA/CC. SOCIALES EN LA LOMCE Y LOE

\begin{tabular}{|c|c|c|c|c|}
\hline \multirow{2}{*}{$\begin{array}{l}\text { Etapas } \\
\text { del SEE }\end{array}$} & \multicolumn{2}{|r|}{$L O E$} & \multicolumn{2}{|c|}{ LOMCE } \\
\hline & Curso & Denominación & Curso & Denominación \\
\hline \multirow{2}{*}{$\begin{array}{c}\text { FORMACIÓN } \\
\text { PROFESIONAL } \\
\text { BÁSICA }\end{array}$} & & & $2^{\circ}$ Curso & $\begin{array}{l}\text { Ciencias } \\
\text { Sociales }\end{array}$ \\
\hline & & & $1^{\circ}$ Curso & Ciencias Sociales \\
\hline BACHILLERATO & $\begin{array}{c}2^{\circ} \text { Curso } \\
\text { (Modalidad de } \\
\text { Humanidades y } \\
\text { CC. Sociales) }\end{array}$ & Geografía & $\begin{array}{c}2^{\circ} \text { Curso } \\
\text { (Modalidad de } \\
\text { Humanidades y } \\
\text { CC. Sociales) }\end{array}$ & Geografía \\
\hline \multirow{2}{*}{ E.S.O. } & $4^{\circ}$ & $\begin{array}{c}\text { Ciencias Sociales, } \\
\text { Geografía e Historia }^{6}\end{array}$ & $2^{\circ} \operatorname{Ciclo}\left(4^{\circ}\right)$ & $\begin{array}{c}\text { Geografía e } \\
\text { Historia }\end{array}$ \\
\hline & $1^{\circ}$ a $3^{\circ}$ & $\begin{array}{c}\text { Ciencias Sociales, } \\
\text { Geografía e Historia }\end{array}$ & $\begin{array}{c}\mathbf{1}^{\mathbf{0}} \text { Ciclo } \\
\left(1^{\circ}, 2^{\circ} \text { y } 3^{\circ}\right) \\
\end{array}$ & $\begin{array}{c}\text { Geografía e } \\
\text { Historia } \\
\end{array}$ \\
\hline $\begin{array}{l}\text { EDUCACIÓN } \\
\text { PRIMARIA }\end{array}$ & $1^{\circ}$ a $6^{\circ}$ & $\begin{array}{c}\text { Conocimiento del } \\
\text { medio natural, social } \\
\text { y cultural }\end{array}$ & $1^{\circ}$ a $6^{\circ}$ & Ciencias Sociales \\
\hline
\end{tabular}

Fuente: LOMCE y LOE. Elaboración propia.

5. En el Bachillerato se da una coincidencia total tanto en cursos y edad $\left(1^{\circ}\right.$ y $2^{\circ}$ para 16-18 años) como en modalidades (Ciencias; Artes; y Humanidades y CC. Sociales) (artículo 34 de LOMCE y articulo 26,4 del RD 1105/2014), con la única diferencia de que en la LOMCE se denomina a esta la modalidad «Ciencias» y en la LOE se denominaba «Ciencias y Tecnología».

Las asignaturas en esta etapa están clasificadas en troncales y específicas (articulo 27 del RD 1105/2014), y, dentro de las troncales, diferencia entre generales y de opción. Ofrece distintos itinerarios dentro de dos de las modalidades que ofrece. Así, en la modalidad de Ciencias ofrece (artículo 34bis), de una forma un tanto encubierta, a través de la elección de

6 Aunque se imparten Ciencias Sociales en el currículo está dedicado a Historia Contemporánea, y por tanto no incluye conocimientos geográficos. 
asignaturas en primer curso, dos itinerarios: uno que podríamos llamar Tecnológico, porque la ley no les da ningún nombre, si se elige Dibujo Técnico, y otro que se podría denominar de Ciencias de la Naturaleza; y en la modalidad de Humanidades y Ciencias Sociales, ofrece otras dos modalidades, en esta ocasión con especificación, que son: el de Humanidades y el Ciencias Sociales ${ }^{7}$.

En el segundo curso se sigue la misma estructura que en el primero en cuanto a modalidades y opciones que, en el caso de la Geografía, se ofrece como una asignatura troncal de opción con este nombre y a elegir, junto con otra, entre un total de cinco (Economía de la empresa, Geografía, Griego II, Historia del Arte e historia de la Filosofía). Es decir, sigue en una situación muy semejante a como está en la $\mathrm{LOE}^{8}$.

Al final de la etapa se contempla una prueba de evaluación final en la que sí hay comprobación de adquisición de competencias en Geografía en el caso de que haya sido elegida (artículo 26 bis y artículos 30 y 31 del RD 1105/2014). Con la superación de esta evaluación se está en condiciones de obtener el título de Bachiller y acceder a la Universidad (Cuadro 4).

6. La Formación Profesional en la LOMCE está organizada en: a) Ciclos de Formación Profesional Básica; b) Ciclos formativos de grado medio; y c) Ciclos formativos de grado superior (artículo 39), mientras que en la LOE solo se contemplaban los ciclos de grado medio y de grado superior. A nosotros, desde el punto de vista de la Geografía, nos interesa solo el primer tipo de ciclo -Formación Profesional Básica- ya que es en el único donde se contempla la enseñanza de las Ciencias Sociales dentro uno de los dos bloques de materias que en él se determinan. Concretamente en el Bloque de Comunicación y Ciencias Sociales ${ }^{9}$. La duración prevista para el ciclo es de dos años por lo que las Ciencias Sociales se han de impartir en esos dos cursos. en relación a la evaluación

7. Uno de los aspectos mas novedosos de la LOMCE, en su conjunto y que por tanto afecta a la Geografía, es la inclusión de los estándares de aprendizaje evaluables en el currículum que se definen como «especificaciones de los criterios de evaluación que permiten definir los resultados de aprendizaje, y que concretan lo que el alumno debe saber, comprender y saber hacer en cada asignatura. Además, el mismo Real Decreto

7 2. En la modalidad de Humanidades y Ciencias Sociales, los alumnos y alumnas deben cursar las siguientes materias generales del bloque de asignaturas troncales: a) Filosofía; b) Lengua Castellana y Literatura I; c) Primera Lengua Extranjera I; d) Para el itinerario de Humanidades, Latín I. Para el itinerario de Ciencias Sociales, Matemáticas Aplicadas a las Ciencias Sociales I (artículo 27 del RD 1105/2014).

8 Sociales, Matemáticas Aplicadas a las Ciencias Sociales II.

e) En función de la regulación y de la programación de la oferta educativa que establezca cada Administración educativa y, en su caso, de la oferta de los centros docentes, al menos dos materias de entre las siguientes materias de opción del bloque de asignaturas troncales, organizadas, en su caso, en bloques que faciliten el tránsito a la educación superior: $1 .^{\circ}$ Economía de la Empresa; $2 .^{\circ}$ Geografía; $3 .^{\circ}$ Griego II; 4. ${ }^{\circ}$ Historia del Arte; $5 .^{\circ}$ Historia de la Filosofía (artículo 28 del RD 1105/2014).

9 «Artículo 42. Contenido y organización de la oferta.

competencias necesarias para su desarrollo profesional, personal y social.

4. Los ciclos de Formación Profesional Básica garantizarán la adquisición de las competencias del aprendizaje permanente a través de la impartición de enseñanzas organizadas en los siguientes bloques comunes:

a) Bloque de Comunicación y Ciencias Sociales, que incluirá las siguientes materias: $1^{\circ}$ Lengua Castellana; $2^{\circ}$ Lengua extranjera; $3^{\circ}$ Ciencias Sociales; $4 .^{\circ}$ En su caso, Lengua Cooficial. 
establece las características que deben tener: deben ser observables, medibles y evaluables y permitir graduar el rendimiento o logro alcanzado». El propio currículum define su utilidad: a) Definir los resultados del aprendizaje; y b) Concretar lo que el alumno debe saber, comprender y saber hacer. Es decir, los estándares de aprendizaje evaluables son la referencia más concreta y objetiva del qué enseñar y del qué evaluar.

La diferencia entre los estándares de aprendizaje evaluables y los criterios de evaluación, está en que aquellos son una referencia más concreta, deben de ser observables, medibles y sobre todo, son una graduación para la consecución del criterio de evaluación. De aquí que los estándares de aprendizaje evaluables están referidos a los criterios de evaluación, o, lo que es igual, de cada criterio de evaluación se deducen una serie de estándares de aprendizaje evaluables.

Los estándares de aprendizaje evaluables se encuentran en el currículum oficial y están definidos en cada uno de los RDs (concretamente en los anexos de los mismos) que desarrollan la LOMCE y cada comunidad autónoma los define, de manera concreta, en los decretos que sobre el currículum desarrolla. Por ejemplo para Educación Primaria en el caso de la Comunidad de Castilla-La Mancha la distribución es la siguiente: $1^{\circ}$. Cada asignatura está secuenciada por cursos; $2^{\circ}$. En cada curso, la asignatura se divide en bloques de contenido; y $3^{\circ}$. En cada bloque de contenido se establecen los contenidos, los criterios de evaluación y los estándares de aprendizaje evaluables de cada uno de los criterios de evaluación.

\section{III.2. La Geografía en los RDs, una concreción de los currículos básicos}

Para el desarrollo de la LOMCE se han publicado los siguientes RDs:

a) El RD 126/2014 (de 28 de febrero por el que se establece el currículo básico de Educación Primaria) sustituye al RD 1513/2006, de 7 de diciembre, por el que se establecen las enseñanzas mínimas de la Educación primaria.

b) El RD 127/2014, de 28 de febrero, por el que se regulan aspectos específicos de la Formación Profesional Básica de las enseñanzas de formación profesional del SEE, se aprueban catorce títulos profesionales básicos, se fijan sus currículos básicos y se modifica el RD 1850/2009, de 4 de diciembre, sobre expedición de títulos académicos y profesionales correspondientes a las enseñanzas establecidas en la Ley Orgánica 2/2006, de 3 de mayo, de Educación.

c) Y por último, el RD 1105/2014, de 26 de diciembre en el que se establece el currículo de la E.S.O. y Bachillerato modifica al RD 1467/2007, de 2 de noviembre, y en él se establece la estructura de la ESO y Bachillerato a la vez que se fijan sus enseñanzas mínimas en la nueva ley.

En la elaboración de los mismos hay que destacar la intensa actividad de la AGE a través de diferentes reuniones con el Gabinete de la Secretaria de Estado de Educación, Formación Profesional y Universidades realizando propuestas concretas a los borradores presentados.

El contenido de estos RDs hacen referencia a las competencias del Estado en materia educativa, tienen el carácter de norma básica y se dicta al amparo del artículo 149.1.30ª de la 
Constitución, que atribuye al Estado las competencias para la regulación de las condiciones de obtención, expedición y homologación de los títulos académicos y profesionales y normas básicas para el desarrollo del artículo 27 de la Constitución, a fin de garantizar el cumplimiento de las obligaciones de los poderes públicos en esta materia.

\section{a) Los conocimientos geográficos en la Educación Primaria}

Los conocimientos geográficos en la etapa de Primaria, según se comentó antes, están incluidos en el área de Ciencias Sociales, dado que el Conocimiento del Medio se ha desdoblado en las áreas de Ciencias de la Naturaleza y Ciencias Sociales, y esta nueva área está incluida dentro de las áreas troncales (Cuadro 5).

Según la LOMCE, en las Ciencias Sociales se integran diversas disciplinas que estudian a las personas como seres sociales y su realidad en sus aspectos geográficos, sociológicos, económicos e históricos. El objeto de las Ciencias Sociales en esta etapa es aprender a vivir en sociedad, conociendo los mecanismos fundamentales de la democracia y respetando las reglas de la vida colectiva.

Cuadro 5

LOS BLOQUES TEMÁTICOS DEL CURRÍCULO BÁSICO DE GEOGRAFÍA EN PRIMARIA

\begin{tabular}{|l|l|}
\hline \multicolumn{1}{|c|}{ LOMCE } & \multicolumn{1}{c|}{ LOE } \\
\hline \multicolumn{1}{|c|}{ PRIMARIA: Ciencias Sociales } & \multicolumn{1}{c|}{ PRIMARIA: } \\
Conocimiento del medio natural, social y cultural \\
\hline Bloque 1: Contenidos comunes & Bloque 1. El entorno y su conservación \\
Bloque 2: EI mundo en que vivimos & Bloque 2. La diversidad de los seres vivos \\
Bloque 3: Vivir en Sociedad & Bloque 3. La salud y el desarrollo personal \\
& Bloque 4. Personas, culturas y organización social \\
& Bloque 5. Cambios en el tiempo \\
& Bloque 6. Materia y energía \\
& Bloque 7. Objetos, máquinas y tecnologías \\
\hline
\end{tabular}

Fuente: RD 126/2014 de la LOMCE y RD 1513/2006 de la LOE. Elaboración propia.

Las críticas más significativas que ha recibido este cambio han sido: la recentralización del currículo; por la evaluación dada la importancia que se le ha dado a los contenidos, a los Criterios de evaluación y a los Estándares de aprendizaje evaluables, frente a los objetivos y competencias específicas que desarrolla cada una de estas materias (Bolívar, A. 2014); y por la «desintegración» de Conocimiento del Medio en dos áreas distintas.

Desde nuestro punto de vista, consideramos que en la LOMCE se ha producido un avance en relación al tratamiento de la Geografía en esta etapa educativa, basado en los siguientes puntos:

1. Consideramos interesante el hecho de que en el currículo de Primaria se separe Conocimiento del Medio natural del medio social, por cuanto contribuye a que la geografía 
tenga un papel significativo dentro de la materia de Ciencias Sociales, materia que a su vez tiene un carácter troncal en toda la Educación Primaria (artículo 4). Actuación que, por otra parte, responde a las pautas dadas en la Declaración Internacional sobre Educación Geográfica, redactada por la Comisión de Educación Geográfica de la Unión Geográfica Internacional en 1992. Pauta que, sin embargo, había sido obviada. Coincidimos con De Miguel González (2012:17) en que un referente tan destacado de esa Declaración Internacional debería haber sido tenido en cuenta en cualquier proceso de revisión curricular de la Geografía.

2. El hecho de que los conocimientos geográficos se incluyan en un área más específica (CC. Sociales), lo consideramos positivo por cuanto supera con ello una de las críticas que habitualmente se habían hecho a la LOE en relación a la Geografía por estar inserta en el área de Conocimiento del Medio Natural, Social y Cultural, que es un área muy amplia que «integra contenidos muy diversos que le otorgan un carácter heterogéneo por lo que se pierde la unidad de cada uno de los contenidos que la integran, entre ellas la Geografía. Esta situación crea al alumnado dificultades en el tránsito a E.S.O., donde se van a encontrar con materias diferentes para lo que antes era un contenido común. Sin embargo, y en favor de esta agrupación actual, hay que decir, que en el trabajo por competencias, es más efectivo si se realiza desde ámbitos amplios de conocimiento que desde materias aisladas» (Buzo Sánchez, I, 2012:38).

3. Como consecuencia, los contenidos geográficos en la LOMCE son más precisos y más geográficos, aspecto que se puede acreditar tanto con la comparación del enunciado del conjunto de bloques de contenidos en ambos RDs (Cuadro 5), como en el desglose de algunos de dichos bloques (Cuadro 6). En concreto los bloques $n^{\circ} 2$ y 3 de la LOMCE y los bloques $n^{\circ} 1$ y 4 de la LOE.

4. Con la nueva ubicación y el nuevo enunciado de los conocimientos geográficos en la LOMCE se facilita al alumno la puesta en relación de los conocimientos geográficos adquiridos en Primaria con los que ha de adquirir en la E.S.O. (Geografía e Historia) y en el Bachillerato (Geografía).

Sin embargo, existen algunos aspectos pendientes de precisar que podrán mejorar o empeorar la situación de nuestra disciplina. Nos referimos a que los cuatro bloques de contenidos (Cuadro 5) en que se organizan las Ciencias Sociales en el RD 126/2014 se han de impartir a lo largo de los seis años que dura la etapa sin que haya ninguna determinación de cómo han de distribuirse por cursos; ni como se han de compaginar con los contenidos de Historia; ni si el aprendizaje se ha de realizar de forma cíclica para ir profundizando cada vez más en ellos o si se ha de estudiar cada bloque una sola vez en la etapa y, por tanto, adaptado a la edad que tenga el alumno en ese momento; ni el horario semanal que se ha de dedicar a esta área.

Estos aspectos, que serán abordados por las respectivas CC. AA. al desarrollar el contenido de este RD en función de sus competencias y teniendo en cuenta lo que, con bastante insistencia, se dice en el texto legislativo que comentamos, sobre la autonomía de los centros y por ende de los departamentos de la disciplina. 


\section{Cuadro 6 \\ COMPARACIÓN DEL CONTENIDO DE ALGUNOS BLOQUES TEMÁTICOS DEL CURRÍCULO BÁSICO DE GEOGRAFÍA EN PRIMARIA (LOMCE Y LOE)}

\begin{tabular}{|c|c|}
\hline LOMCE & LOE \\
\hline Bloque 2: El mundo en que vivimos & Bloque 1. El entorno y su conservación \\
\hline 1. El Universo y el Sistema Solar: La Tierra & $\begin{array}{l}\text { - Orientación de elementos del medio físico en } \\
\text { relación con el sol. }\end{array}$ \\
\hline $\begin{array}{l}\text { 2. La representación de la Tierra. } \\
\text { Orientación en el espacio y Coordenadas }\end{array}$ & $\begin{array}{l}\text { - Percepción y descripción de algunos elementos y } \\
\text { fenómenos naturales: la luna, las estrellas y el sol, } \\
\text { el día y la noche. }\end{array}$ \\
\hline 3. La Atmosfera. El tiempo atmosférico & $\begin{array}{l}\text { - Observación de algunos fenómenos atmosféricos } \\
\text { y su representación. }\end{array}$ \\
\hline 4. El clima & $\begin{array}{l}\text { - Elementos básicos del medio físico: el aire y } \\
\text { el agua. Uso responsable del agua en la vida } \\
\text { cotidiana. }\end{array}$ \\
\hline 5. La hidrosfera: El agua & $\begin{array}{l}\text { - Observación, exploración e inicio de sencillos } \\
\text { trabajos de algún ecosistema concreto, acuático o } \\
\text { terrestre. }\end{array}$ \\
\hline 6. La litosfera: las rocas y el relieve & $\begin{array}{l}\text { - Observación y percepción de algunos elementos } \\
\text { naturales y humanos del entorno. }\end{array}$ \\
\hline 7.El Paisaje: Elementos del paisaje & Bloque 4. Personas, culturas y organización social \\
\hline 8. La Intervención Humana en el Medio. & $\begin{array}{l}\text { - La familia. Relaciones entre sus miembros. } \\
\text { Reparto equilibrado de las tareas domésticas y } \\
\text { adquisición de responsabilidades. }\end{array}$ \\
\hline 9. El desarrollo sostenible & $\begin{array}{l}\text { - Principales tareas y responsabilidades de los } \\
\text { miembros de la comunidad educativa. Valoración } \\
\text { de la participación de todos. }\end{array}$ \\
\hline Bloque 3: Vivir en Sociedad & $\begin{array}{l}\text { - Conciencia de los derechos y deberes de las } \\
\text { personas en el grupo. }\end{array}$ \\
\hline $\begin{array}{l}\text { 1. La Organización social política y } \\
\text { territorial del Estado español. }\end{array}$ & $\begin{array}{l}\text { - Simulación de situaciones y conflictos de } \\
\text { convivencia. }\end{array}$ \\
\hline 2. La Población & $\begin{array}{l}\text { - Acercamiento a las manifestaciones de las } \\
\text { culturas presentes en el entorno, como muestra de } \\
\text { diversidad y riqueza. }\end{array}$ \\
\hline 3. Economía. Los sectores de producción & $\begin{array}{l}\text { - Reconocimiento de diferentes profesiones sin } \\
\text { estereotipos sexistas. }\end{array}$ \\
\hline $\begin{array}{l}\text { 4. La empresa. La vida económica de los } \\
\text { Ciudadanos. Educación Financiera }\end{array}$ & $\begin{array}{l}\text { - Formas de organización en el entorno próximo: } \\
\text { la escuela y el municipio. Introducción al } \\
\text { conocimiento de las responsabilidades y tareas de } \\
\text { las instituciones locales. }\end{array}$ \\
\hline 5. Educación Vial & \\
\hline
\end{tabular}

Fuente: RD 126/2014 de la LOMCE y RD 1513/2006 de la LOE. Elaboración propia. 


\section{b) Los conocimientos geográficos en la E.S.O.}

La E.S.O. se regula en la LOMCE en los artículos comprendidos entre el 23bis y el 29, y en el RD 1105/2014 (del artículo 10 al 23) se incluye, entre las asignaturas Troncales, una denominada Geografía e Historia, denominación que supone una especificación positiva para nuestra disciplina dentro del conjunto de CC. Sociales que tenía en Educación Primaria, a la vez que se identifica con la asignatura de Geografía que se contempla en el Bachillerato.

Uno de los aspectos positivos que se han observado entre los borradores y la Ley orgánica aprobada respecto a las enseñanzas de la materia de Ciencias Sociales, Geografía e Historia es que en cuarto de la ESO, en el anteproyecto dejaba de ser obligatoria para todos los alumnos, para pasar a ser una materia optativa vinculada a la modalidad. Mientras que en la actualidad se recoge como materia obligatoria para todos los estudiantes de cuarto, independientemente de su modalidad. Crítica que ya se lanzó en el foro del I Congreso Europeo de Didáctica de la Geografía, celebrado en Zaragoza en de noviembre 2012, y que también fue recogida y expuesta por la AGE a las instancias educativas pertinentes.

En el RD 1105/2014, publicado el 3 de enero de 2015, encontramos algunos aspectos que no han cambiado muchas de las deficiencias detectadas y expresadas:

1. No se ha mejorado la delimitación por cursos de los contenidos geográficos que había en la LOE, donde comparte su tiempo con la Historia durante los dos primeros cursos, queda independiente en tercero y no se estudia nada en cuarto. Pese a las críticas que se habían hecho a esta estructura de la LOE desde distintas instancias profesionales (AGE especialmente), en la LOMCE se sigue con la misma confusión (AGE, 2014:4).

2. El currículo de la ESO se vincula principal, pero no exclusivamente, en lo que respecta a Geografía e Historia a dos competencias: social y ciudadana, y conocimiento e interacción con el mundo físico. No obstante, la ESO en el RD 1105/2014 no hace una especial contribución de la materia a la adquisición de la competencia digital, sin embargo en Europa hay un referente que es esencial a la hora de afrontar la renovación curricular de la Geografía: la Agenda Digital Europea (2010) ha establecido la alfabetización y la adquisición en competencias digitales como acciones claves y prioritarias para el reglamento del Fondo Social Europeo, así como el hecho de identificar las competencias de los profesionales y usuarios en el marco de las Técnicas de Información y Comunicación (en adelante TIC) en relación con el citado Marco Europeo de Cualificaciones. Ello tiene unas implicaciones evidentes en el caso de la educación geográfica, especialmente en el uso de la información geográfica en las aulas (Miguel González, 2012: 20).

3. Tampoco se ha cambiado la «indisoluble unidad» de la Geografía con la Historia que se daba en la E.S.O. de la LOE, tanto desde el punto de vista docente como curricular, mezcla que en los dos primeros cursos daba lugar, en ocasiones, a que los conocimientos geográficos fuesen relegados a un segundo plano, debido a factores como la extensión de los temarios de Historia que en muchas ocasiones impiden desarrollar el contenido geográfico con el tiempo adecuado; la mayor especialización del profesorado hacia la Historia, por lo que se centran sus objetivos más hacia el estudio 
temporal de las sociedades que al espacial; o la inclusión de contenidos geográficos en otras (Buzo Sánchez, I, 2012: 38). Esta situación, según viene expresada en el RD $1105 / 2014$, se mantiene en la LOMCE puesto que no hay especificación si esos bloques se van a impartir uno en cada uno de los tres cursos del primer ciclo o si se van a compartir en cada uno de los tres cursos del primer ciclo de E.S.O.

4. El tipo de conocimientos geográficos que se «proponen resultan poco conformes a la evolución científica de la Geografía en nuestro días y bastante ajenos a los enfoques conceptuales y metodológicos que vienen orientando a la Geografía desde hace varias décadas desde una perspectiva académica. A título de ejemplo se pueden señalar algunos aspectos preocupantes por la falta de actualidad de los conceptos subyacentes como el de paisaje, entendido aquí exclusivamente como paisaje natural sin tener en cuenta las manifestaciones culturales (Convenio de Florencia, 2000). Si bien esta carencia se corrige en $2^{\circ}$ de Bachillerato. Lo mismo ocurre con el entendimiento de las actividades agrarias, energéticas, industriales y su localización territorial así como los procesos de urbanización y globalización, o la disminución de los contenidos referidos a los usos y actividades económicas en los contenidos geográficos de las asignaturas de todos los cursos de E.S.O.» (AGE, 2014:4).

\begin{tabular}{|c|c|}
\hline $\begin{array}{c}\text { LOE } \\
\text { Primer curso (Conocimiento del Medio) }\end{array}$ & $\begin{array}{c}\text { LOMCE } \\
\text { Geografía e Historia }\left(1^{\circ}\right. \\
\text { Ciclo ESO) }\end{array}$ \\
\hline $\begin{array}{l}\text { Bloque 1. Contenidos comunes } \\
\text { Bloque 2. La Tierra y los medios naturales } \\
\text { Bloque } 3 \text {. Sociedades prehistóricas, } 1^{\circ} \text { civilizaciones y E. Antigua. }\end{array}$ & $\begin{array}{l}\text { Bloque 1: } \text { El medio físico } \\
\text { 1.1.- La Tierra } \\
\text { 1.2.- España }\end{array}$ \\
\hline Segundo curso (Conocimiento del Medio) & 1.3.- Europa \\
\hline $\begin{array}{l}\text { Bloque 1. Contenidos comunes } \\
\text { Bloque 2. Población y sociedad. } \\
\text { Bloque 3. Las sociedades preindustriales }\end{array}$ & $\begin{array}{l}\text { 1.4.- Mundial } \\
\text { Bloque 2: El espacio humano } \\
\text { 2.1.- España }\end{array}$ \\
\hline Tercer curso (Conocimiento del Medio) & 2.2.- Europa \\
\hline $\begin{array}{l}\text { Bloque 1. Contenidos comunes } \\
\text { Bloque 2. Actividad económica y espacio geográfico } \\
\text { Bloque 3. Organización política y espacio geográfico. } \\
\text { Bloque 4. Transformaciones y desequilibrios en el mundo actual. }\end{array}$ & $\begin{array}{l}\text { 2.3.- Mundial } \\
\text { Bloque } 3 \text { : La Historia }\end{array}$ \\
\hline Cuarto curso (Conocimiento del Medio) & $\begin{array}{c}\text { Geografía e Historia }\left(2^{\circ}\right. \\
\left.\text { Ciclo ESO: } 4^{\circ}\right)\end{array}$ \\
\hline $\begin{array}{l}\text { Bloque 1. Contenidos comunes. } \\
\text { Bloque 2. Bases históricas de la sociedad actual. } \\
\text { Bloque 3. El mundo actual. }\end{array}$ & $\begin{array}{l}\text { Todos los bloques o temas son } \\
\text { de Historia contemporánea }\end{array}$ \\
\hline
\end{tabular}

Fuente: LOMCE, RD 1105/2014 y RD 1631/2006. Elaboración propia. 
5. Los bloques temáticos que se proponen (cuadro 7) tienen, desde nuestro punto de vista, y coincidiendo plenamente con la alegación $n^{\circ} 3$ de la AGE (2014:4-5), un carácter muy repetitivo en sus contenidos ya que «se reproducen a diferentes escalas (España-Europa-Mundo) los mismos contenidos. Se continúa con el esquema clásico de Geografía física-Geografía humana-Geografía regional de los tres últimos currículos, con cambios más bien en su organización, distribución o vinculación a contenidos procedimentales y actitudinales. Aunque es cierto que en el segundo y en el tercer curso se incluyen desde la LOE más contenidos transversales a las ciencias sociales y a su función de educación ciudadana (Miguel González, 2012: 15).

Podemos concluir afirmando que muchas de las críticas que se habían hecho a la LOE no aparecen corregidas o superadas en la LOMCE y en los RDs que la desarrollan.

Nuestra propuesta de concreción será que el bloque 1 sobre el medio físico podía impartirse en $1^{\circ}$ curso y el bloque 2 sobre el espacio humano en $2^{\circ}$ curso, dejando el bloque 3 , que es de Historia, para el $3^{\circ}$ curso. Y la de dejar la asignatura de cuarto de ESO (Geografía e Historia), como una materia de currículo integrado, esto es, como una Geografía para el Mundo Contemporáneo que partiera de unos antecedentes históricos básicos, especialmente desde la segunda mitad del siglo pasado. Esta sería una asignatura que explicaría perfectamente los grandes retos, problemas y desequilibrios del mundo actual, incluyendo buena parte de los temas expuestos hace dos párrafos, así como contenidos del currículo actual en los cursos de tercero y cuarto.

Sin olvidar, que la revisión curricular de la Geografía tiene un reto pendiente, al margen de la evolución epistemológica de la ciencia geográfica referente, que es la innovación en educación geográfica donde la nueva orientación didáctica europea de educación en competencias, con especial relevancia de la competencia espacial, digital y social (Miguel González, 2012: 21).

\section{c) Los conocimientos geográficos en el Bachillerato}

En el caso del Bachillerato, la ordenación académica de la Geografía no cambia, aunque tampoco se ha hecho una revisión curricular, lo que supone una ocasión perdida de revisión, adaptación y mejora.

En cuanto a los contenidos de esta disciplina (Cuadro $n^{\circ} 8$ ) se centran en una Geografía de España en relación con el espacio europeo y mundial y se les ha dado una mayor especificación en la LOMCE que los que tenía en la LOE y, entendemos, que un orden más lógico, aunque más clásico. Si bien es cierto que se dedica un primer bloque, con carácter introductorio, a la ciencia geográfica, al territorio, al paisaje y a las técnicas cartográficas, que es muy positivo, por cuanto se amplían y concretan los mismos con respecto a la LOE.

Sirva de ejemplo la comparación entre el bloque 3 de la LOE y la especificación de los mismos contenidos en los bloques 2, 3, 4 y 5 de la LOMCE.

En Bachillerato, tanto en la LOE como en la LOMCE, se ha de partir, en la práctica, de cero. Así la parte de Geografía Física en la LOE no se ve con cierta profundidad desde $1^{\circ}$ de la E.S.O. (cuatro años antes) y en la LOMCE vuelve a suceder lo mismo. Situación que da lugar a que a los alumnos esta materia se les haga muy complicada y difícil de estudiar. 


\section{LOS BLOQUES TEMÁTICOS DE GEOGRAFÍA EN BACHILLERATO (LOMCE Y LOE)}

\begin{tabular}{|c|c|}
\hline LOMCE & LOE \\
\hline $\begin{array}{l}\text { Bloque } 1 \text {. La geografía y el estudio del espacio } \\
\text { geográfico }\end{array}$ & 1. Contenidos comunes \\
\hline $\begin{array}{l}\text { Bloque 2. El relieve español, su diversidad } \\
\text { geomorfológica }\end{array}$ & 2. España en Europa y en el mundo \\
\hline Bloque 3. La diversidad climática y la vegetación & 3. Naturaleza y medio ambiente en España \\
\hline Bloque 4. La hidrografía & 4. Territorio y actividades económicas en España \\
\hline $\begin{array}{l}\text { Bloque 5. Los paisajes naturales y las } \\
\text { interrelaciones naturaleza-sociedad }\end{array}$ & $\begin{array}{l}\text { 5. Población, sistema urbano y contrastes } \\
\text { regionales en España }\end{array}$ \\
\hline Bloque 6. La población española & \\
\hline $\begin{array}{l}\text { Bloque } 7 . \text { El espacio rural y las actividades del } \\
\text { sector primario }\end{array}$ & \\
\hline $\begin{array}{l}\text { Bloque } 8 \text {. Las fuentes de energía y el espacio } \\
\text { industrial }\end{array}$ & \\
\hline Bloque 9. El sector servicios & \\
\hline Bloque 10. El espacio urbano & \\
\hline Bloque 11. Formas de organización territorial & \\
\hline Bloque 12. España en Europa y en el mundo & \\
\hline
\end{tabular}

Fuente: LOMCE, RD 1105/2014 y RD 1467/2007. Elaboración propia.

Por otra parte, si no se rectifica esta distribución la mayoría de los alumnos que opten por el Bachillerato de Humanidades y Ciencias Sociales en la LOMCE, al igual que les ocurría a los de la LOE, no habrán estudiado contenidos de Geología o de Medio Ambiente en sus materias optativas de $4^{\circ}$ de la E.S.O., pues, generalmente, aunque están dentro de las optativas de ese curso, no suelen ser elegidas por los alumnos que se orientan a un Bachillerato de Humanidades y CC. Sociales. Por lo que respecta a la parte de $\mathrm{G}^{\mathrm{a}}$ Humana y Económica se les hace más llevadera, aunque hayan pasado también algunos años desde su estudio en $3^{\circ}$ de la E.S.O. (Valenzuela et al, 2004).

La existencia de las Pruebas de Acceso a la Universidad (PAU) en la LOE suponía una espada de Damocles sobre el profesor que imparte docencia en $2^{\circ}$ de Bachillerato en materias que son objeto de examen en esa prueba, como es el caso de la Geografía. La extensión del temario y la obligatoriedad de impartirlo en su totalidad y en profundidad, puesto que el alumno va a examinarse de todo al final del curso, dificulta la labor del profesorado que no puede dedicar demasiado tiempo a cada contenido, ni utilizar metodologías didácticas más prácticas, ni introducir de manera continuada en las clases las TIG, hecho clave para mejorar la cada vez más necesaria competencia espacial.

Sin embargo, la existencia de esta misma prueba, asegura una homogeneidad de contenidos impartidos en cada distrito universitario, que de otra forma no se conseguiría (Buzo Sánchez, 2012: 39). En la LOMCE (artículos 30-31 del RD 1105/2014) se mantiene de hecho esta misma prueba (Prueba Final de Etapa) y por tanto la misma presión aunque, sin embargo, no garantice de forma automática el acceso a la Universidad 


\section{d) Los conocimientos geográficos en la Formación Profesional}

Una de las principales novedades de la LOMCE son los títulos de Formación Profesional Básica dirigida a alumnos que hayan cursado el $1^{\circ}$ ciclo de la ESO y que no están en condiciones de promocionar al $4^{\circ}$ curso (o, excepcionalmente, al $3^{\circ}$ curso) y se accede a propuesta del equipo docente o consejo orientador. El Título obtenido al cursar un ciclo de Formación Profesional Básica tiene validez académica y profesional a la vez que permite el acceso directo a la Formación Profesional de Grado Medio, tiene los mismos efectos laborales (no académicos) que el título de Graduado en ESO para acceder a empleos públicos y privados y permite presentarse a la prueba de evaluación final de la ESO (reválida) y, en caso de superarla, obtener el Graduado en ESO por la opción correspondiente.

Estos títulos se desarrollan en el RD 127/2014 y tendrán la misma estructura que el resto de títulos de las enseñanzas de Formación Profesional del sistema educativo. En ellos se incluirán los siguientes módulos profesionales:

1. Módulos asociados a unidades de competencia del Catálogo Nacional de Cualificaciones Profesionales.

2. Módulos asociados a los bloques comunes, que garantizarán la adquisición de las competencias del aprendizaje permanente:

2.1. Módulo de Comunicación y Sociedad I y Módulo de Comunicación y Sociedad II, en los que se desarrollan competencias del bloque común de Comunicación y Ciencias Sociales, que incluyen las siguientes materias:1. ' Lengua castellana;

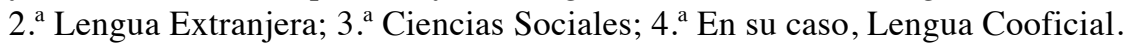

2.2. Módulo de Ciencias Aplicadas I y Ciencias Aplicadas II, en los que se desarrollan competencias de las materias del bloque común de Ciencias Aplicadas, que incluye las siguientes materias: $1^{\mathrm{a}}$ Matemáticas Aplicadas al Contexto Personal y de Aprendizaje de un Campo Profesional; 2a Ciencias Aplicadas al Contexto Personal y de Aprendizaje de un Campo Profesional.

3. Módulo de formación en centros de trabajo.

La presencia de la geografía se garantiza dentro de los módulos comunes a través de la materia de CC. Sociales, concretamente en el Bloque de Comunicación y Ciencias Sociales, en el que se prevé que se impartan Ciencias Sociales en los dos cursos que forman el ciclo. Entendemos que esto supone un avance para nuestra disciplina, ya que la está ofertado a todos aquellos alumnos que opten por estos ciclos.

\section{PERPETUACIÓN DE LAS CARENCIAS Y OMISIÓN PARCIAL DE LAS PROPUESTAS DE LOS PROFESIONALES. UNA OCASIÓN PERDIDA}

Decíamos al comienzo de este artículo que una reforma legislativa de carácter educativo abre expectativas de cambio en los profesionales de cada una de las áreas de conocimiento. Se pretende que se recoja en ella el estado actual de los conocimientos del área correspondiente, la toma en consideración de los planteamientos didácticos que se hayan producido hasta el momento y hayan demostrado su eficacia, y que su encuadre en el sistema permita alcanzar los objetivos educativos que le son propios. 
En consecuencia, entendemos que debemos, en primer lugar, señalar las carencias detectadas en la norma que se reforma (en nuestro caso la LOE); conocer las propuestas que se han hecho para conseguir la mejora, especialmente las propuestas de la Asociación de Geógrafos Españoles (AGE); y valorar en qué medida se han rectificado esas carencias y/o se han tomado en consideración las propuestas formuladas en la nueva norma (LOMCE).

\section{IV.1. Las carencias y deficiencias de los contenidos geográficos en la LOE como punto de partida para valorar su situación en la LOMCE}

Un aspecto que consideramos fundamental en este análisis comparativo que estamos realizando es determinar en qué medida la situación y el tratamiento que tienen los conocimientos geográficos en la vigente LOE están necesitados de cambios y mejoras para, en base a ellos, poder valorar, de un lado, las propuestas de mejora que se han hecho desde las aportaciones de distintos profesionales y en especial de la AGE; y, de otro, conocer si se han rectificado, en todo o en parte, en la nueva ley (LOMCE).

Sintetizando mucho de lo expuesto, siguiendo a Buzo (2012), las carencias más destacadas son:

1. Los contenidos de Geografía e Historia que se proponen no siempre están debidamente ajustados al tiempo disponible, sobre todo cuando ambas disciplinas se han de impartir en el mismo curso ( $1^{\circ}$ y $2^{\circ}$ de E.S.O.), de forma que la amplitud de los temarios de Historia en muchas ocasiones impide tratar con la suficiente profundidad los contenidos indicados para Geografía. A lo que contribuye el hecho de que la formación inicial del profesorado (especialidad) es predominantemente en Historia y esa situación da un sesgo importante a la forma de trabajar la materia.

2. Los contenidos más complejos de entender, como es el caso de la Geografía Física, están localizados en los cursos más bajos ( $1^{\circ}$ de E.S.O.), es decir, cuando menos capacidad de comprensión tienen los alumnos.

3. Una gran discontinuidad en los contenidos geográficos en la etapa de E.S.O. y Bachillerato, especialmente para los alumnos que cursan el Bachillerato de Humanidades y Ciencias Sociales. Efectivamente se estudia Geografía en los tres primeros cursos de E.S.O. pero en $4^{\circ}$ y $1^{\circ}$ de Bachillerato no hay ningún contenido geográfico, que no se vuelven a retomar hasta $2^{\circ}$ de Bachillerato, vacío que repercute considerablemente en la materia

4. Los contenidos de la Geografía de $2^{\circ}$ de Bachillerato en la modalidad de Humanidades y Ciencias Sociales es muy extenso, máxime cuando el profesor y el alumno están presionados por la PAU, de ahí la necesidad de reflexionar y consensuar sobre todo entre la Universidad y los Institutos unos contenidos más ajustados a la formación inicial que traen los alumnos de la E.S.O. y a las posibilidades temporales de un curso.

5. No se tiene en cuenta, de forma satisfactoria, los valores educativos de la Geografía en la formación básica de todos los ciudadanos (AGE, 2014:1). No podemos perder de vista que la enseñanza de la Geografía, por requerir una constante trasposición 
didáctica de los problemas actuales del mundo y de los desafíos sociales (ambientales, demográficos, urbanísticos, socioeconómicos), que tienen una dimensión territorial, en un contexto complejo, cambiante y global (Miguel González, R., 2012:152), puede contribuir de forma eficaz, ya sea directamente o como materia transversal, a conseguir ese objetivo formativo (AGE, 2014:8-9).

\section{IV.2. Las propuestas de mejora de los contenidos geográficos realizadas por la Asociación de Geógrafos Españoles durante la gestación de la LOMCE}

La preocupación de la AGE por la situación en que podría quedar la Geografía en la LOMCE se ha puesto de manifiesto de forma continuada desde que se anunció el proyecto de reforma por parte del Gobierno, hasta la publicación de los RDs del currículo básico, siendo sus hitos más importantes los siguientes:

1. Reunión con la Secretaria de Estado de Educación, Formación Profesional y Universidades el 7 de mayo de 2012 en la que los representantes de la AGE expusieron sus preocupaciones con respecto a la presencia de la Geografía en la Educación Secundaria ante las reformas del Sistema Educativo y su ofrecimiento de colaboración y asesoramiento al Ministerio de Educación en temas de diseño curricular y contenidos de las materias vinculadas con la Geografía.

2. Envío de un escrito al Ministerio de Educación mostrando su preocupación sobre la supresión de la materia de Ciencias Sociales de $4^{\circ}$ de la E.S.O. y su transformación en optativa exclusivamente en la modalidad académica.

3. Realización de una encuesta entre los profesores de Geografía e Historia de educación Secundaria para conocer su opinión sobre los posibles cambios que se pudieran introducir para la mejora de la enseñanza de la Geografía en la Educación Secundaria.

4. Publicación en 2013 de un informe sobre «La posición de la Geografía en la Educación Secundaria y el Bachillerato», donde se recogen los resultados de la encuesta que se había realizado entre los profesores de Geografía e Historia durante el curso 2012/13 por la AGE y contestada por más de 600 profesores de Geografía de todo el Estado. Dicho informe se remitió al Ministerio en octubre de 2013.

5. Publicación y remisión al MEDC, a fines del 2013, del Análisis del Proyecto de Real Decreto por el que se establece el currículo básico de la Educación Primaria, de la E.S.O. y del Bachillerato en lo que compete a las asignaturas de Geografía y Propuestas de la (AGE)

6. Envío de los borradores a los socios y otros profesores para recoger sus impresiones y realizar las alegaciones oportunas.

7. Elaboración de un documento con las alegaciones recogidas publicado en la web de la AGE, que se envió al Ministerio en el periodo de revisión del proyecto.

8. Entrevista de la directiva de la AGE con el Asesor del Gabinete de la Secretaria de Estado de Educación, Formación Profesional y Universidades y en estos momentos Director General de Evaluación y Cooperación Territorial), el 27 de diciembre de 2013. 
9. Nuevos contactos con socios de la AGE y otros profesores de Secundaria y Universidad para realizar propuestas concretas al curriculum.

10. Reunión e informe con propuestas concretas al borrador presentado para el curriculum de Primaria por parte directiva de la AGE a finales de enero de 2014 que se envió posteriormente al Ministerio.

11. Reunión e informe con propuestas concretas al borrador presentado para el curriculum de la ESO y Bachillerato por parte directiva de la AGE a finales de febrero de 2014 que se envió posteriormente al Ministerio.

Las propuestas de mejora que ha hecho la AGE, como se comentó antes, han estado en gran medida condicionadas por las propuestas oficiales, es decir, no han sido propuestas hechas por ella en función de como entendía que debía tratarse disciplina, sino meras sugerencias de cambio a las propuestas del MECD. No obstante es de valorar el esfuerzo y los resultados obtenidos por este colectivo. Y que, de forma resumida, señalamos a continuación, agrupados en cuatro bloques: curriculares, de apoyo a las TIC, de apoyo al plurilingüismo, y en la formación del profesorado (Cuadro 9).

Cuadro 9

SÍNTESIS DE LAS PROPUESTAS DE MEJORA ELABORADAS POR LAASOCIACIÓN DE GEÓGRAFOS ESPAÑOLES

\begin{tabular}{|l|l|}
\hline \multicolumn{1}{|c|}{ Bloque de Propuestas } & \multicolumn{1}{c|}{ Concreción de propuestas } \\
\hline \multirow{2}{*}{ Propuestas curriculares } & En cuanto al plan de estudios \\
\cline { 2 - 3 } & En cuanto a contenidos \\
\hline $\begin{array}{l}\text { Propuestas de apoyo a las } \\
\text { Técnicas de Información } \\
\text { y Comunicación (TIC) }\end{array}$ & $\begin{array}{l}\text { Incorporarlo en el Curriculum el estudio y uso de las TIC en } \\
\text { Geografía Apoyo y docencia de materias optativas relacionadas }\end{array}$ \\
\hline $\begin{array}{l}\text { Propuestas de apoyo al } \\
\text { plurilingüismo }\end{array}$ & El dominio de la segunda lengua (profesor y alumno) \\
\cline { 2 - 2 } & No reducir los contenidos de Geografía por dificultades de idioma \\
\hline $\begin{array}{l}\text { Propuestas en la } \\
\text { formación del profesorado }\end{array}$ & $\begin{array}{l}\text { Pruebas de acceso a la función pública (Historia, Arte y Geografía) no } \\
\text { específicas de Geografía. Escasa formación didáctica en el Grado }\end{array}$ \\
\hline
\end{tabular}

Fuente: Secretaría de la Asociación de Geógrafos Españoles. Elaboración propia.

\section{a) Propuestas curriculares}

\section{- En cuanto al plan de estudios}

- Reestructurar el plan de estudios general para redistribuir la materia en la E.S.O. y Bachillerato y lograr mantener la continuidad de los contenidos geográficos en ambas etapas salvando el hueco de dos años que se da en la actualidad ( $4^{\circ}$ de E.S.O. y $1^{\circ}$ de Bachillerato).

- Ampliar la presencia de la Geografía en la Educación Primaria, E.S.O. y Bachillerato. En el primer caso sería conveniente, de una parte, que, al estar repartido el tiempo 
con la enseñanza de la Historia y ser el contenido de ambas muy amplio, determinar el tiempo que se ha de destinar a cada una de las materias; y de otro adaptar de forma realista los contenidos de ambas. En el segundo caso, bien añadiendo una materia de Geografía en $1^{\circ}$ de Bachillerato o bien incorporando contenidos de Geografía Política a la Historia del Mundo Contemporáneo de $1^{\circ}$ de Bachillerato, o agrupando los contenidos geográficos que aparecen dispersos en los cursos de $1^{\circ}$ y $2^{\circ}$ de la E.S.O. en un único curso de Geografía.

- Incluir alguna materia de Geografía como obligatoria para todos los itinerarios de Bachillerato (ya sea en $1^{\circ} \mathrm{o}$ en $2^{\circ} \mathrm{o}$ en los dos).

- Ampliar la optatividad con materias vinculadas a la Geografía tanto en $1^{\circ}$ como en $2^{\circ}$. Optatividad que puede ser de ampliación (Geografía Física, Humana, etc...) como de técnicas geográficas (TIG, prácticas, trabajo de campo, etc.), y ello tanto en itinerarios de Humanidades y CC. SS. como en los Científicos-tecnológicos.

- Incorporación de módulos de formación geográfica, impartidos por profesores de la especialidad, en determinados Ciclos Formativos ya existentes (relacionados con el Turismo, Actividades en el medio natural, etc.) o algún nuevo ciclo formativo relacionado con las TIG.

- En cuanto a contenidos

- Precisar, acotar y coordinar los contenidos de la Geografía con respecto a otras materias del currículo para evitar repeticiones (caso, a título de ejemplo, de las Ciencias Naturales y la Geografía Física, o eliminar referencias históricas en muchos de los temas evitando redundancias con otras materias del currículo)

- Ordenar la disposición y nivel de profundidad de los contenidos que se imparten en E.S.O. con respecto a los de Bachillerato para que no haya necesidad de repetir esos mismos contenidos en cursos posteriores de esa misma etapa o en otras etapas.

- Incluir contenidos de Geografía Mundial Actual en $4^{\circ}$ de la E.S.O., complementando la Historia del Mundo Contemporáneo. Contenidos que pueden ir en la misma materia o en otra materia autónoma.

- Evitar la redundancia que se produce en los contenidos de la Geografía de $2^{\circ}$ de Bachillerato al estudiar lo mismo a distintas escalas (por ejemplo, población general de España primero y autonómica después), por lo que se deberían centrar los contenidos en una sola escala.

- Evitar la dispersión de escala que se da en la Geografía de $2^{\circ}$ de Bachillerato (Mundo, Europa, España, Comunidades Autónomas) para lo que sería conveniente bien centrarse solo en España, eliminando referencias superiores (Europa, Mundo) e inferiores (CC.AA); o bien centrase en el mundo y, en todo caso, analizar la relación de España en el mundo.

- Dar un enfoque más práctico (tales como análisis demográficos, económicos, cartográficos) y relacionado con las TIG (SIG, Teledetección, Geoposicionamiento, etc.), con metodologías activas, evitando enfoques academicistas y mostrando su utilidad para resolver problemas. 


\section{b) Propuestas de apoyo a las TIC}

Las TIC es uno de los objetivos de la LOMCE (BOE $n^{\circ} 295$, de 10 diciembre 2013, Sec. I. Pág. 97865, punto XI del preámbulo): «La incorporación generalizada al sistema educativo de las TIC, que tendrán en cuenta los principios de diseño para todas las personas y accesibilidad universal, permitirá personalizar la educación y adaptarla a las necesidades y al ritmo de cada alumno o alumna.».

Entendemos que la Geografía puede jugar un papel muy importante al contar con herramientas tecnológicas propias. Su uso debería integrarse claramente en el currículo, mediante dos vías: como objeto de estudio en sí y como herramienta para aprender Geografía. Su aprendizaje también podría estar incorporado a las materias optativas relacionadas con las TIC, que pudieran ser impartidas por los profesores de Geografía.

No obstante existen algunas dificultades prácticas que conviene tener presentes con el fin de tratar de superarlas, tales como el que los ordenadores disponibles en los centros son escasos o están obsoletos, el elevado número de alumnos por aula, falta de formación adecuada del profesorado y que los programas informáticos son complejos y necesitan unos ordenadores de cierta capacidad y un adiestramiento específico para su manejo.

\section{c) Propuestas de apoyo al plurilingüismo}

Otro de los objetivos que se propone la LOMCE es el plurilingüismo: «El dominio de una segunda o, incluso, una tercera lengua extranjera se ha convertido en una prioridad en la educación como consecuencia del proceso de globalización en que vivimos, a la vez que se muestra como una de las principales carencias de nuestro sistema educativo. La Unión Europea fija el fomento del plurilingüismo como un objetivo irrenunciable para la construcción de un proyecto europeo» (BOE $\mathrm{n}^{\circ}$ 295, de 10 diciembre 2013, Sec. I. Pág. 97865, punto XII del preámbulo). Junto a él, en la enseñanza de los idiomas cada vez se ha generalizado más la utilización de otras materias ajenas al propio idioma como instrumento de aprendizaje.

En esta línea, las Ciencias Sociales y con ellas la Geografía puede ser una de ellas, si bien, desde nuestro punto de vista, es necesario: 1) El dominio de la segunda lengua por parte del profesor y del alumno; 2) que no se reduzcan los contenidos de Geografía previstos para un determinado curso en base a las dificultades de la enseñanza y el aprendizaje en otro idioma, porque eso supondría disminuir la formación específica en Geografía, aunque se potenciase el del plurilingüismo.

\section{d) Propuestas en la formación del profesorado}

Uno de los problemas actuales en la formación del profesorado es que su formación inicial se ha hecho a partir de una Licenciatura bien en Historia, bien en Geografía, o bien en Arte, salvo raras excepciones que han cursado dos o tres de esas licenciaturas, lo que resulta insuficiente y provoca carencias a la hora de ejercer la docencia dado que en el ejercicio profesional se han de impartir las tres materias. Esta carencia inicial se ve, en parte, compensada, con la preparación de las pruebas que han de realizar para acceder a la función pública docente en las que se incluyen contenidos de las tres materias. 
Otro de los problemas del profesorado actual es la falta de preparación didáctica en su currículo formativo, ya que la formación de este tipo que recibe es de carácter postgradual, es decir, en los tres meses de prácticas en el aula que se contemplan en el Master de Formación del Profesorado si bien supone un gran avance con respecto al anterior Curso de Adaptación Pedagógica (CAP).

De estas consideraciones se deduce que la formación del profesorado que ha de enseñar Geografía no siempre es la adecuada, aunque igual podría comentarse en relación a la enseñanza de la Historia por los licenciados en Geografía. Ante estas dos realidades es necesario diseñar una formación inicial y continua del profesorado que le capacite y actualice adecuadamente para el ejercicio de su función docente.

Entre las múltiples soluciones que se aportan en la encuesta de la AGE nos inclinamos por unos estudios de Grado en los que se incluyan las tres materias que, posteriormente, se van a impartir como docente, y ello incorporando a esa formación básica complementos formativos en los Master de Formación del Profesorado para aquellos alumnos que no sean de la especialidad (Geografía para Historia e Historia del Arte y viceversa), así como de TIG.

En cuanto a la formación didáctica caben varias opciones: ofrecer un itinerario formativo dirigido a la docencia desde las titulaciones de Grado; organización de una formación práctica en los centros docentes, al estilo del MIR en sanidad.

\section{IV.3. Las carencias de la LOMCE en relación a los contenidos geográficos ¿Una ocasión perdida?}

La nueva ley LOMCE y los RDs que la desarrollan, no han recogido, ni mucho menos, las propuestas que se le han hecho desde la AGE en relación a los conocimientos geográficos en la Enseñanza Primaria, E.S.O. y Bachillerato, por lo que entendemos que ha sido, en gran medida, una ocasión perdida para haber subsanado las carencias que ya existían en la LOE.

Los cambios que se han producido en la LOMCE en relación a los conocimientos geográficos los podemos sintetizar en los siguientes puntos:

\section{a) En relación a las etapas y cursos}

La LOMCE con respecto a la LOE, en lo que hace a etapas y cursos, no ha generado cambios sustanciales (cuadro 4). No obstante podemos indicar los siguientes (Buzo Sánchez, I, 2012):

- Se ha producido un cambio en cuanto a la ubicación de área de conocimiento y en cuanto a su denominación. Así, en lo que respecta a su ubicación, en la Enseñanza Primaria propuesta por la LOMCE se crea un área de conocimiento específica, denominada Ciencias Sociales, cómo área independiente, mientras que en la LOE las ciencias sociales estaban incluidas en el área de Conocimiento del medio natural, social y cultural, es decir, unida a las Ciencias Naturales.

Este cambio está generando una profunda polémica abundando los argumentos en pro y en contra: para unos, la inclusión en el área del medio natural permite presentar el conocimiento de la realidad como algo global, múltiple e interrelacionado, que el alumno, de forma 
gradual, irá diferenciando. Además, argumentan, el trabajo por competencias es más efectivo desde ámbitos de conocimiento más que desde materias aisladas. Para otros, la gran heterogeneidad de contenidos que se integran en el área de Conocimiento del Medio da lugar a que se pierda esa unidad de la materia y crear una cierta confusión en el alumno ya que, cuando llega a cursar la E.S.O., esos conocimientos diversos aparecen separados en distintas disciplinas y, en el caso concreto de los conocimientos geográficos, se produce una separación entre los contenidos físicos y humanos.

Por tanto, consideran positivo que desde la Enseñanza Primaria hasta el Bachillerato se les denomine como Ciencias Sociales, o Geografía e Historia, aunque habría que matizar que esta uniformidad no es tan perfecta por cuanto en Educación Primaria se les llama Ciencias Sociales, en E.S.O. Geografía e Historia, en Bachillerato se mantiene esa misma denominación y en Formación Profesional Básica se vuelve al término Ciencias Sociales.

- Se mantiene, en principio, el mismo peso de los conocimientos geográficos en las distintas etapas del nuevo SEE aunque se haya producido su separación en Ciencias Sociales en Primaria, pero se mantiene en la E.S.O. y Bachillerato.

\section{b) En relación a los contenidos}

- En los currículos propuestos por los RDs, ya sean publicados o en proyecto, «hay ausencia prácticamente absoluta de contenidos de Geografía regional, tanto autonómica como local, y de una geografía exterior a la Unión Europea. En el contexto actual se considera fundamental enseñar España en su diversidad regional, histórica, autonómica, incluso nacional, es decir explicar una geografía regional renovada, actual, dinámica y vivida. Lo mismo respecto al ámbito mundial, no puede entenderse que los estudiantes de enseñanzas preuniversitarias no estudien nada sobre África, América, el continente asiático, o sobre los BRICS (Brasil, Rusia, India, China y Sudáfrica). Son, en consecuencia, programas que adolecen de un alto grado de etnocentrismo, hispano centrismo y eurocentrismo» (AGE, alegación 7, p 5-6), si bien cabe que en la parte del currículo que han de diseñar las CC.AA. se subsane esta carencia.

- «No existe una clara delimitación por cursos de los contenidos geográficos del primer ciclo de E.S.O. si bien podría pensarse que en el tercer curso en la asignatura de Geografía e Historia se compartirían contenidos de Geografía e Historia Moderna. La Geografía queda aquí reducida a un mero complemento de los estudios históricos, sin que exista tampoco ninguna articulación e integración entre los contenidos temáticos de unos y otros en cada uno de los cursos» (AGE, alegación 1, pág. 4).

- Los contenidos geográficos que se proponen, al igual que ocurría en la LOE, «resultan poco conformes a la evolución científica de la Geografía en nuestro días y bastante ajenos a los enfoques conceptuales y metodológicos que vienen orientando a la Geografía desde hace varias décadas desde una perspectiva académica. A título de ejemplo se pueden señalar algunos aspectos preocupantes por la falta de actualidad de los conceptos subyacentes como el de paisaje, entendido aquí exclusivamente como paisaje natural sin tener en cuenta las manifestaciones culturales (Convenio de Florencia, 2000). Lo mismo ocurre con el entendimiento de las actividades agrarias, 
energéticas, industriales y su localización territorial así como los procesos de urbanización y globalización, o la disminución de los contenidos referidos a los usos y actividades económicas en los contenidos geográficos de las asignaturas de todos los cursos de E.S.O.» (AGE, alegación 2, pág. 4).

- Se vuelve a repetir en la LOMCE el planteamiento de la LOE con respecto a «los contenidos de una Geografía descriptiva, prácticamente desechada hace muchos años en todos los niveles educativos por su obsolescencia e ineficacia formativa, que apenas considera las interrelaciones entre esos diversos componentes. Así, el hecho de comenzar el estudio por la Geografía de España, sin que el alumnado disponga de las herramientas conceptuales básicas para comprender los hechos espaciales y sin integrar su evolución y características en el contexto mundial y europeo, supondrá una visión excesivamente descriptiva y «localista» carente, además, de articulación con los contenidos históricos de la misma asignatura» (AGE, alegación 3, pág. 5).

- Una revisión curricular, tanto en la ESO como en el Bachillerato, debe ser capaz de integrar aquellos temas sociales y territoriales de mayor actualidad, de tal manera que la enseñanza de la Geografía escolar pueda ser considerada como una materia de interés para el alumnado, con contenidos actualizados para la comprensión de los fenómenos sociales contemporáneos o de los problemas del mundo (García Pérez, 2011).

\section{CONCLUSIÓN}

Una reforma educativa, como se ha dicho ya, es una buena ocasión para corregir y mejorar las deficiencias que se detecten en el sistema en general y de cada disciplina en particular. Son precisamente los profesionales de una determinada materia no solo los más interesados sino los más capacitados para proponer, después de las oportunas encuestas y debates, los que formulen, en amplio y profundo consenso, las propuestas que consideran más convenientes.

De la misma forma entendemos que los legisladores y la Administración educativa han de apoyarse en estas propuestas de los profesionales para establecer el desarrollo curricular en las respectivas materias escolares, superando y conjugando los intereses particularistas de cada grupo y atendiendo al bien general.

Estos dos principios, desde nuestro punto de vista, no se han tenido suficientemente en cuenta a la hora de plantear la Geografía en la LOMCE ya que no sufre cambios importantes en lo que a etapas y cursos se refiere con respecto a la situación que tiene en la vigente LOE, ni tampoco se mejoran, en aspectos sustanciales, los contenidos, adaptándolos a los avances de nuestra disciplina por lo que consideramos que ha sido una ocasión perdida que, no obstante, sería aconsejable y deseable que se corrijan las deficiencias actuales, hasta donde sea posible, en el desarrollo que las CC.AA hagan de los RDs. De lo contrario perpetuará los errores y las carencias, y prescindirá, lamentablemente, de las ricas y abundantes aportaciones que los conocimientos geográficos pueden proporcionar en la formación integral de las personas.

\section{AGRADECIMIENTOS}

Quisiera agradecer a los revisores anónimos de este artículo sus consejos y aportaciones. Las cuáles han contribuido a mejorar el trabajo presentado. 


\section{REFERENCIAS}

ASOCIACIÓN DE GEÓGRAFOS ESPAÑOLES (AGE) (2014): Alegaciones AGE al proyecto de R.D sobre los currículos de ESO y del Bachillerato. Análisis y alegaciones de la AGE al Proyecto de Real Decreto sobre los currículos de Educación Secundaria Obligatoria y del Bachillerato. Edición on-line: http://www.age-geografia.es/gestion/uploads/ Alega_AGE_RD_Educacion_DEF.pdf (Fecha de consulta: 12/02/2014)

BOLÍVAR, A. (2014): «Los nuevos currículos de la LOMCE. Un primer análisis crítico» en Escuela, $\mathrm{n}^{\circ} 4007$ (68).

BUZO SÁNCHEZ, I. (2012): «La posición de los contenidos geográficos en la Reforma Educativa LOMCE» en Miguel González, R., De Lázaro y Torres, M.L. y Marrón Gaite, M.J. (Eds.): La educación geográfica digital, Grupo de Didáctica de la Geografía de la Asociación de Geógrafos Españoles y Universidad de Zaragoza, en colaboración con la red Comenius digitalearth.eu, pp. 37- 48.

BUZO SÁNCHEZ, I. e IBARRA BENLLOCH, P. (2013): Informe: La posición de la Geografía en la Educación Secundaria y el Bachillerato. Ed. Asociación de Geógrafos Españoles. AGE. Edición on-line: http://www.age-geografia.es/gestion/uploads/Informe_ AGE.pdf (Fecha de consulta: 12/02/2014)

GARCÍA PÉREZ, F. (2011): «Geografía, problemas sociales y conocimiento escolar». Anekumene. Vol 1, n ${ }^{\circ}$ 2, pp. 6-21.

GONZÁLEZ GALLEGO, I. (2001): «La formación inicial y permanente del profesorado de Geografía: una necesidad y un reto en el momento actual», en Marrón, M.J. (Ed.) La formación geográfica de los ciudadanos en el cambio de milenio. Madrid: y Universidad Complutense de Madrid, pp. 673-70.

GONZÁLEZ GALLEGO, I. (2011): «Análisis crítico de las opciones curriculares en la educación secundaria obligatoria», en Prats, J. (Coord.) Geografía e Historia. Complementos de formación disciplinar. Barcelona: Graó, pp. 161-186.

LUIS GÓMEZ, A. y ROMERO, J. (2006): «Expectativas sociales, racionalidades educativas y reformulación de la función social del conocimiento geográfico en la escuela anglosajona tras la Segunda Guerra Mundial: del enciclopedismo culturalista al academicismo cognitivo», Didáctica Geográfica, segunda época, nº 7, pp. 331-363.

MIGUEL GONZÁLEZ, R. DE (2012): «Análisis comparativo del currículo de Geografía en Educación Secundaria y propuestas didácticas», en Miguel González, R., De Lázaro y Torres, M M L. y Marrón Gaite, M.J. (Eds.): La educación geográfica digital, Grupo de Didáctica de la Geografía de la Asociación de Geógrafos Españoles y Universidad de Zaragoza, en colaboración con la red Comenius digitalearth.eu, pp. 13-36.

RODRÍGUEZ DOMÉNECH, M.A. (2008): «Una enseñanza nueva en una cultura nueva. El caso de la Geografía en el Bachillerato». Diez años de cambios en el Mundo, en la Geografía y en las Ciencias Sociales, 1999-2008. Actas del X Coloquio Internacional de Geocrítica, Universidad de Barcelona, 26-30 de mayo de 2008. http://www.ub.es/ geocrit/-xcol/314.htm (Fecha de consulta: 12/02/2014)

SOUTO, X.M. (2003): «Las reformas escolares y la Geografía en el umbral del siglo XXI», en Marrón, M.J., Moraleda, C. y Rodríguez, H. (Eds.) La enseñanza de la Geografía ante 
las nuevas demandas sociales. Toledo: Grupo de Didáctica de la Geografía (AGE). Universidad de Castilla-La Mancha. Escuela Universitaria del Profesorado.

SOUTO, X.M. (2004): «La Geografía escolar en el periodo 1990-2003», en La Geografía española ante los retos de la sociedad actual. Aportación española al XXX. Congreso de la UGI. Glasgow, Comité Español de la UGI 2004, pp. 61-82.

SOUTO, X.M. (2011): «Fines y objetivos en la enseñanza de la Geografía: los condicionantes sociales y epistemológcos», en Prats, J. (Coord.) Didáctica de la Geografía e Historia. Barcelona, Graó, pp. 115-129.

VALENZUELA, M., DE LÁZARO, M.L. y MOLLÁ, M. (2004): «Geography in Spain. The diffusion of geographical thought and its place in civil society». Belgeo 1, pp. 143-161.

\section{NORMATIVA}

LEY ORGÁNICA 8/2013, de 9 de diciembre, para la mejora de la calidad educativa, BOE, núm. 295, martes 10 de diciembre de 2013, Sec. I. pág. 97858. Edición on-line: http:// www.boe.es/boe/dias/2013/12/10/pdfs/BOE-A-2013-12886.pdf (Fecha de consulta: $12 / 02 / 2014)$

MECD: Propuestas para el anteproyecto de Ley Orgánica para la Mejora de la Calidad Educativa. LOMCE. Fecha de creación: 11/06/2012. Edición on-line:http://www.mecd.gob. es/servicios-al-ciudadano-mecd/dms/mecd/servicios-al-ciudadano-mecd/participacionpublica/lomce/propuestas-anteproyecto-24072012.pdf (Fecha de consulta: 12/02/2014)

MECD: Primer borrador de la LOMCE. Fecha de creación: 25/09/2012. Edición online:https:// docs.google.com/file/d/0Bx29VZJ6ZfEDTk9zNVhBaFVxTnM/edit?usp=sharing\&pli=1 (Fecha de consulta: 12/02/2014)

MECD: Segundo borrador de la LOMCE. Fecha de creación: 3/12/2012. Edición online:https://docs.google.com/file/d/0Bx29VZJ6ZfEDOENicU1 odldLR1E/ edit?usp=sharing\&pli=1(Fecha de consulta: 12/02/2014)

MECD: Tercer borrador de la LOMCE. Fecha de creación: 14/02/2013. Edición online:https://docs.google.com/file/d/0Bx29VZJ6ZfEDZ0hiZWpiN3JSd3c/ edit?usp=sharing\&pli=1(Fecha de consulta: 12/02/2014)

MECD: Cuarto borrador de la LOMCE publicado en el Boletín Oficial de las Cortes Generales como: 121/000048. Proyecto de Ley Orgánica para la mejora de la calidad educativa enviado a las Cortes Generales del Congreso de los Diputados. Fecha de creación: 8/10/2013. Edición on line: http://www.congrE.S.O..es/public_oficiales/L10/CONG/ BOCG/A/BOCG-10-A-48-4.PDF (Fecha de consulta: 12/02/2014)

REAL DECRETO 126/2014, de 28 de febrero, por el que se establece el currículo básico de la Educación Primaria y se fijan sus enseñanzas mínimas. BOE $\mathrm{n}^{\circ} 52$, martes 1 marzo 2014. pág. 19349 Edición on line: http://www.boe.es/boe/dias/2014/03/01/pdfs/BOE-A2014-2222.pdf (Fecha de consulta: 16/05/2014)

REAL DECRETO 127/2014, de 28 de febrero, por el que se establece el currículo básico de la Formación Profesional Básica y se fijan sus enseñanzas mínimas. BOE n ${ }^{\circ} 55$, martes 4 marzo 2014. pág. 20155. Edición on line: https://www.boe.es/boe/dias/2014/03/05/pdfs/ BOE-A-2014-2360.pdf (Fecha de consulta: 16/05/2014) 
REAL DECRETO 1467/2007, de 2 de noviembre, por el que se establece la estructura del Bachillerato y se fijan sus enseñanzas mínimas. BOE n ${ }^{\circ}$ 266, martes 6 noviembre 2007. pág. 45381. Edición on line: http://www.boe.es/boe/dias/2007/11/06/pdfs/A4538145477.pdf (Fecha de consulta: 12/02/2014)

REAL DECRETO 1513/2006, de 7 de diciembre, por el que se establecen las enseñanzas mínimas de la Educación Primaria. BOE n 293 Viernes 8 diciembre 2006, pág. 43053, Edición on line: http://www.boe.es/boe/dias/2006/12/08/pdfs/A43053-43102.pdf (Fecha de consulta: 16/05/2014)

REAL DECRETO 1631/2006, de 29 de diciembre, por el que se establecen las enseñanzas mínimas correspondientes a la Educación Secundaria Obligatoria. BOE $\mathrm{n}^{\mathrm{o}} 5$, viernes 5 enero 2007, pág. 677. Edición on line: http://www.boe.es/diario_boe/txt.php?id=BOEA-2007-238 (Fecha de consulta: 12/02/2014)

REAL DECRETO 126/2014, de 28 de febrero, por el que se establece el currículo básico de Educación Primaria, BOE n ${ }^{\circ}$ 52, sábado 1 de marzo 2014, pág. 19349, Edición on line: www.boe.es/boe/dias/2014/03/01/pdfs/BOE-A-2014-2222.pdf.

REAL DECRETO 127/2014, de 28 de febrero, por el que se regulan aspectos específicos de la Formación Profesional Básica de las enseñanzas de formación profesional del sistema educativo, se aprueban catorce títulos profesionales básicos, se fijan sus currículos básicos y se modifica el Real Decreto 1850/2009, de 4 de diciembre, sobre expedición de títulos académicos y profesionales correspondientes a las enseñanzas establecidas en la Ley Orgánica 2/2006, de 3 de mayo, de Educación, BOE n 55, miércoles, 5 de marzo de 2014, pág. 20155, Edición on line: https://www.boe.es/boe/dias/2014/03/05/pdfs/BOEA-2014-2360.pdf.

REAL DECRETO 1105/2014, de 26 de diciembre, por el que se establece el currículo de la E.S.O. y Bachillerato, BOE ${ }^{\circ}$ 3, 3 de enero de 2015, pág. 169, Edición on line: https:// www.boe.es/boe/dias/2015/01/03/pdfs/BOE-A-2015-37.pdf 
